\title{
Averaging Principles for Nonautonomous Two-Time-Scale Stochastic Reaction-Diffusion Equations with Jump
}

\author{
Yong $X u \mathbb{D}^{1,2}$ and Ruifang Wang ${ }^{1}$ \\ ${ }^{1}$ Department of Applied Mathematics, Northwestern Polytechnical University, Xi'an 710072, China \\ ${ }^{2}$ MIIT Key Laboratory of Dynamics and Control of Complex Systems, Northwestern Polytechnical University, \\ Xi'an 710072, China
}

Correspondence should be addressed to Yong Xu; hsux3@nwpu.edu.cn

Received 9 January 2020; Revised 7 July 2020; Accepted 14 July 2020; Published 19 September 2020

Academic Editor: Eulalia Martínez

Copyright (C) 2020 Yong Xu and Ruifang Wang. This is an open access article distributed under the Creative Commons Attribution License, which permits unrestricted use, distribution, and reproduction in any medium, provided the original work is properly cited.

In this paper, we aim to develop the averaging principle for a slow-fast system of stochastic reaction-diffusion equations driven by Poisson random measures. The coefficients of the equation are assumed to be functions of time, and some of them are periodic or almost periodic. Therefore, the Poisson term needs to be processed, and a new averaged equation needs to be given. For this reason, the existence of time-dependent evolution family of measures associated with the fast equation is studied and proved that it is almost periodic. Next, according to the characteristics of almost periodic functions, the averaged coefficient is defined by the evolution family of measures, and the averaged equation is given. Finally, the validity of the averaging principle is verified by using the Khasminskii method.

\section{Introduction}

The slow-fast systems are widely encountered in biology, ecology, and other application areas. In this paper, we are concerned with the following nonautonomous slow-fast systems of stochastic partial differential equations (SPDEs) on a bounded domain $\mathcal{O}$ of $\mathbb{R}^{d}(d \geq 1)$ :

$$
\left\{\begin{array}{l}
\frac{\partial u_{\epsilon}}{\partial t}(t, \xi)=\mathscr{A}_{1}(t) u_{\epsilon}(t, \xi)+b_{1}\left(t, \xi, u_{\epsilon}(t, \xi), v_{\epsilon}(t, \xi)\right)+f_{1}\left(t, \xi, u_{\epsilon}(t, \xi)\right) \frac{\partial \omega^{Q_{1}}}{\partial t}(t, \xi)+\int_{\mathbb{Z}} g_{1}\left(t, \xi, u_{\epsilon}(t, \xi), z\right) \frac{\partial \tilde{N}_{1}}{\partial t}(t, \xi, d z) \\
\frac{\partial v_{\epsilon}}{\partial t}(t, \xi)=\frac{1}{\epsilon}\left[\left(\mathscr{A}_{2}(t)-\alpha\right) v_{\epsilon}(t, \xi)+b_{2}\left(t, \xi, u_{\epsilon}(t, \xi), v_{\epsilon}(t, \xi)\right)\right]+\frac{1}{\sqrt{\epsilon}} f_{2}\left(t, \xi, u_{\epsilon}(t, \xi), v_{\epsilon}(t, \xi)\right) \frac{\partial \omega^{Q_{2}}}{\partial t}(t, \xi)+\int_{\mathbb{Z}} g_{2}\left(t, \xi, u_{\epsilon}(t, \xi), v_{\epsilon}(t, \xi), z\right) \frac{\partial \widetilde{N}_{2}^{\epsilon}}{\partial t}(t, \xi, \mathrm{d} z) \\
u_{\epsilon}(0, \xi)=x(\xi) \\
v_{\epsilon}(0, \xi)=y(\xi) \\
\xi \in \mathcal{O}, \\
\mathscr{N}_{1} u_{\epsilon}(t, \xi)=\mathscr{N}_{2} v_{\epsilon}(t, \xi)=0, \quad t \geq 0, \xi \in \partial \mathcal{O}
\end{array}\right.
$$


where $\epsilon \ll 1$ is a positive parameter and $\alpha$ is a sufficiently large fixed constant. The operators $\mathcal{N}_{1}$ and $\mathcal{N}_{2}$ are boundary operators. The stochastic perturbations $\omega^{Q_{1}}, \omega^{Q_{2}}$, and $\widetilde{N}_{1}, \widetilde{N}_{2}^{e}$ are mutually independent Wiener processes and Poisson random measures on the same complete stochastic basis $\left(\Omega, \mathscr{F},\left\{\mathscr{F}_{t}\right\}_{t \geq 0}, \mathbb{P}\right)$, which will be described in Section 2. For $i=1,2$, the operator $\mathscr{A}_{i}(t)$ and the functions $b_{i}, f_{i}$, and $g_{i}$ depend on time, and we assume that the operator $\mathscr{A}_{2}(t)$ is periodic and the functions $b_{1}, b_{2}, f_{2}$, and $g_{2}$ are almost periodic.

The goal of this paper is to establish an effective approximation for the slow equation of original system (1) by using the averaging principle. The averaged equation is obtained as follows:

$$
\left\{\begin{array}{l}
\frac{\partial \bar{u}}{\partial t}(t, \xi)=\mathscr{A}_{1}(t) \bar{u}(t, \xi)+\bar{B}_{1}(\bar{u}(t))(\xi)+f_{1}(t, \xi, \bar{u}(t, \xi)) \frac{\partial \omega^{Q_{1}}}{\partial t}(t, \xi)+\int_{\mathbb{Z}} g_{1}(t, \xi, \bar{u}(t, \xi), z) \frac{\partial \widetilde{N}_{1}}{\partial t}(t, \xi, \mathrm{d} z) \\
\bar{u}(0, \xi)=x(\xi), \quad \xi \in \mathcal{O}, \\
\mathcal{N}_{1} \bar{u}(t, \xi)=0, \quad t \geq 0, \xi \in \partial \mathcal{O},
\end{array}\right.
$$

where $\bar{B}_{1}$ is the averaged coefficient, which will be given in equation (6). To demonstrate the validity of the averaging principle, we prove that, for any $T>0$ and $\eta>0$, it yields

$$
\lim _{\epsilon \longrightarrow 0} \mathbb{P}\left(\sup _{t \in[0, T]}\left\|u_{\epsilon}(t)-\bar{u}(t)\right\|_{L^{2}(\mathcal{O})}>\eta\right)=0,
$$

where $\bar{u}$ is the solution of the averaged equation (2).

The theory of the averaging principle, originated by Laplace and Lagrange, has been applied in celestial mechanics, oscillation theory, radiophysics, and other fields. The firstly rigorous results for the deterministic case were given by Bogolyubov and Mitropolskii [1]. Moreover, Volosov [2] and Besjes [3] also promoted the development of the averaging principle. Then, great interest has appeared in its application to dynamical systems under random perturbations. An important contribution was that, in 1968, Khasminskii [4] originally proposed the averaging principle for stochastic differential equations (SDEs) driven by Brownian motion. Since then, the averaging principle has been an active area of research. Many studies on the averaging principle of SDEs have been presented, e.g, Givon [5], Freidlin and Wentzell [6], Duan [7], Thompson [8], and Xu and his coworkers [9-11]. Recently, effective approximations for slow-fast SPDEs have been received extensive attention. Cerrai $[12,13]$ investigated the validity of the averaging principle for a class of stochastic reaction-diffusion equations with multiplicative noise. In addition, Wang and Roberts [14], Pei and $\mathrm{Xu}$ [15-17], and Li [18] and Gao [19-21] also studied the averaging principles for the slow-fast SPDEs.

The abovementioned works mainly considered autonomous systems. For the autonomous systems, as long as the initial value is given, the solution of which only depends on the duration of time, not on the selection of the initial time. However, if the initial time is different, the solution of nonautonomous equations with the same initial data will also be different. Therefore, compared with the autonomous systems, the dynamic behaviors of the nonautonomous systems are more complex, which can portray more actual models. Chepyzhov and Vishik [22] studied long-time dynamic behaviors of nonautonomous dissipative system. Carvalho [23] dealt with the theory of attractors for the nonautonomous dynamical systems. Bunder and Roberts [24] considered the discrete modelling of nonautonomous PDEs.

In 2017, the averaging principle has been presented for nonautonomous slow-fast system of stochastic reactiondiffusion equations by Cerrai [25]. But, the system of this paper was driven by Gaussian noises, which is considered as an ideal noise source and only can simulate fluctuations near the mean value. Actually, due to the complexity of the external environment, random noise sources encountered in practical fields usually exhibit non-Gaussian properties, which may cause sharp fluctuations. It should be pointed out that Poisson noise, one of the most ubiquitous noise sources in many fields [26-28], can provide an accurate mathematical model to describe discontinuous random processes, some large moves, and unpredictable events [29-31]. So, in this paper, we are devoted to developing the averaging principle for nonautonomous systems of reaction-diffusion equations driven by Wiener processes and Poisson random measures.

The key to using the averaging principle to analyze system (1) is the fast equation with a frozen slow component $x \in L^{2}(\mathcal{O})$ :

$$
\left\{\begin{array}{l}
\frac{\partial v^{x, y}}{\partial t}(t, \xi)=\left[\left(\mathscr{A}_{2}(t)-\alpha\right) v^{x, y}(t, \xi)+b_{2}\left(t, \xi, x(\xi), v^{x, y}(t, \xi)\right)\right]+f_{2}\left(t, \xi, x(\xi), v^{x, y}(t, \xi)\right) \frac{\partial \omega^{Q_{2}}}{\partial t}(t, \xi)+\int_{\mathbb{Z}} g_{2}\left(t, \xi, x(\xi), v^{x, y}(t, \xi), z\right) \frac{\partial \widetilde{N}_{2}^{e}}{\partial t}(t, \xi, \mathrm{d} z) \\
v^{x, y}(s, \xi)=y(\xi), \quad \xi \in \mathcal{O}, \\
\mathcal{N}_{2} v^{x, y}(t, \xi)=0, \quad t \geq s, \xi \in \partial \mathcal{O} .
\end{array}\right.
$$


By dealing with the Poisson terms, we prove that an evolution family of measures $\left(\mu_{t}^{x} ; t \in \mathbb{R}\right)$ on $L^{2}(\mathcal{O})$ for fast equation (4) exists. Then, assuming that $\mathscr{A}_{2}(t)$ is periodic and $b_{2}, f_{2}$, and $g_{2}$ are almost periodic, we prove that the evolution family of measures is almost periodic. Moreover, with the aid of Theorem 2.10 in [32], we prove that the family of functions

$$
\left\{t \in \mathbb{R} \longmapsto \int_{L^{2}(\mathcal{O})} B_{1}(t, x, y) \mu_{t}^{x}(\mathrm{~d} y)\right\},
$$

is uniformly almost periodic for any $x$ in a compact set $\mathbb{K} \subset L^{2}(\mathcal{O})$, where $B_{1}(t, x, y)(\xi)=b_{1}(t, \xi, x(\xi), y(\xi))$ for any $x, y \in L^{2}(\mathcal{O})$ and $\xi \in \mathcal{O}$.

According to the characteristics of almost periodic function ([25], Theorem 3.4), we define the averaged coefficient $\bar{B}_{1}$ as follows:

$$
\bar{B}_{1}(x):=\lim _{T \longrightarrow \infty} \frac{1}{T} \int_{0}^{T} \int_{L^{2}(\mathcal{O})} B_{1}(t, x, y) \mu_{t}^{x}(\mathrm{~d} y) \mathrm{d} t, \quad x \in L^{2}(\mathcal{O}) .
$$

Finally, the averaged equation is obtained through the averaged coefficient $\bar{B}_{1}$. Using the classical Khasminskii method to the present situation, the averaging principle is effective.

The abovementioned notations will be given in Section 2, and in this paper, $c>0$ below with or without subscripts will represent a universal constant whose value may vary in different occasions.

\section{Notations, Assumptions, and Preliminaries}

Let $\mathcal{O}$ be a bounded domain of $\mathbb{R}^{d}(d \geq 1)$ having a smooth boundary. In this paper, we denote $\mathbb{t}$ the separable Hilbert space $L^{2}(\mathcal{O})$, endowed with the usual scalar product,

$$
\langle x, y\rangle_{\boxplus}=\int_{\mathscr{O}} x(\xi) y(\xi) \mathrm{d} \xi,
$$

and with the corresponding norm $\|\cdot\|_{\mathbb{H}}$. The norm in $L^{\infty}(\mathcal{O})$ will be denoted by $\|\cdot\|_{\infty}$.

Furthermore, the subspace $\mathscr{D}\left((-A)^{\theta}\right)$ [33-35] of the generator $A$ is dense in $\mathbb{H}$, and endowed with the norm,

$$
\|\Lambda\|_{\theta}=\left\|(-A)^{\theta} \Lambda\right\|_{\mathbb{\boxplus}}, \quad \Lambda \in \mathscr{D}\left((-A)^{\theta}\right),
$$

for $0 \leq \theta<1,0<t \leq T$. According to Theorem 6.13 in [36], there exists $c_{\theta}>0$, such that

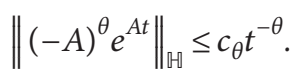

We denote by $B_{b}(\mathbb{W})$ the Banach space of the bounded Borel functions $\varphi: \mathbb{W} \longrightarrow \mathbb{R}$, endowed with the sup-norm

$$
\|\varphi\|_{0}:=\sup _{x \in \boxplus}|\varphi(x)|,
$$

and $C_{b}(\mathbb{H})$ is the subspace of the uniformly continuous mappings. Moreover, $D([s, T] ; \mathbb{U})$ denotes the space of all càdlàg path from $[s, T]$ into $\mathbb{H}$.

We shall denote that $\mathscr{L}(\mathbb{H})$ is the space of the bounded linear operators in $\mathbb{U}$ and denote $\mathscr{L}_{2}(\mathbb{U})$ the subspace of Hilbert-Schmidt operators, endowed with the norm

$$
\|Q\|_{2}=\sqrt{\operatorname{Tr}\left[Q^{*} Q\right]} .
$$

In slow-fast system (1), the Gaussian noises $\partial \omega^{Q_{1}} / \partial t(t, \xi)$ and $\partial \omega^{Q_{2}} / \partial t(t, \xi)$ are assumed to be white in time and colored in space in the case of space dimension $d>1$, for $t \geq 0$ and $\xi \in \mathcal{O}$. And $\omega^{Q_{i}}(t, \xi)(i=1,2)$ is the cylindrical Wiener processes defined as

$$
\omega^{Q_{i}}(t, \xi)=\sum_{k=1}^{\infty} Q_{i} e_{k}(\xi) \beta_{k}(t), \quad i=1,2,
$$

where $\left\{e_{k}\right\}_{k \in \mathbb{N}}$ is a complete orthonormal basis in $\mathbb{H}$, $\left\{\beta_{k}(t)\right\}_{k \in \mathbb{N}}$ is a sequence of mutually independent standard Brownian motion defined on the same complete stochastic basis $\left(\Omega, \mathscr{F},\left\{\mathscr{F}_{t}\right\}_{t \geq 0}, \mathbb{P}\right)$, and $Q_{i}$ is a bounded linear operator on $\mathbb{H}$.

Next, we give the definitions of Poisson random measures $\widetilde{N}_{1}(\mathrm{~d} t, \mathrm{~d} z)$ and $\widetilde{N}_{2}^{\epsilon}(\mathrm{d} t, \mathrm{~d} z)$. Let $(\mathbb{Z}, \mathscr{B}(\mathbb{Z}))$ be a given measurable space and $v(d z)$ be a $\sigma$-finite measure on it. $D_{p_{t}^{i}}, i=1,2$ represents two countable subsets of $\mathbb{R}_{+}$. Moreover, let $p_{t}^{1}, t \in D_{p_{t}^{1}}$ be a stationary $\mathscr{F}_{t}$-adapted Poisson point process on $\mathbb{Z}$ with the characteristic $v$, and $p_{t}^{2}, t \in D_{p_{t}^{2}}$ be the other stationary $\mathscr{F}_{t}$-adapted Poisson point process on $\mathbb{Z}$ with the characteristic $v / \epsilon$. Denote by $N_{i}(\mathrm{~d} t, \mathrm{~d} z), i=1,2$ the Poisson counting measure associated with $p_{t}^{i}$, i.e.,

$$
N_{i}(t, \Lambda):=\sum_{s \in D_{p_{t}^{i}, s \leq t}} I_{\Lambda}\left(p_{t}^{i}\right), \quad i=1,2 .
$$

Let us denote the two independent compensated Poisson measures

$$
\begin{aligned}
& \widetilde{N}_{1}(\mathrm{~d} t, \mathrm{~d} z):=N_{1}(\mathrm{~d} t, \mathrm{~d} z)-v_{1}(\mathrm{~d} z) \mathrm{d} t \\
& \tilde{N}_{2}^{\epsilon}(\mathrm{d} t, \mathrm{~d} z):=N_{2}(\mathrm{~d} t, \mathrm{~d} z)-\frac{1}{\epsilon} v_{2}(\mathrm{~d} z) \mathrm{d} t,
\end{aligned}
$$

where $v_{1}(\mathrm{~d} z) \mathrm{d} t$ and $1 / \epsilon v_{2}(\mathrm{~d} z) \mathrm{d} t$ are the compensators.

Refer to $[28,37]$ for a more detailed description of the stochastic integral with respect to a cylindrical Wiener process and Poisson random measure.

For any $t \in \mathbb{R}$, the operators $\mathscr{A}_{1}(t)$ and $\mathscr{A}_{2}(t)$ are second-order uniformly elliptic operators, having continuous coefficients on $\mathcal{O}$. The operators $\mathcal{N}_{1}$ and $\mathcal{N}_{2}$ are the boundary operators, which can be either the identity operator (Dirichlet boundary condition) or a first-order operator (coefficients satisfying a uniform nontangentiality condition). We shall assume that the operator $\mathscr{A}_{i}(t)$ has the following form:

$$
\mathscr{A}_{i}(t)=\gamma_{i}(t) \mathscr{A}_{i}+\mathscr{L}_{i}(t), \quad t \in \mathbb{R}, i=1,2,
$$

where $\mathscr{A}_{i}$ is a second order uniformly elliptic operator $[38,39]$ with continuous coefficients on $\mathcal{O}$, which is independent of $t$. In addition, $\mathscr{L}_{i}(t)$ is a first-order differential operator, which has the form:

$$
\mathscr{L}_{i}(t, \xi) u(\xi)=\left\langle l_{i}(t, \xi), \nabla u(\xi)\right\rangle_{\mathbb{R}^{d}}, \quad t \in \mathbb{R}, \xi \in \mathcal{O} .
$$

The realizations of the differential operators $\mathscr{A}_{i}$ and $\mathscr{L}_{i}$ in $\mathbb{U}$ are $A_{i}$ and $L_{i}$. Moreover, $A_{1}$ and $A_{2}$ generate two analytic semigroups $e^{t A_{1}}$ and $e^{t A_{2}}$, respectively. 
Now, we give the following assumptions:

(A1)

(a) For $i=1,2$, the function $\gamma_{i}: \mathbb{R} \longrightarrow \mathbb{R}$ is continuous, and there exist $\gamma_{0}, \gamma>0$ such that

$$
\gamma_{0} \leq \gamma_{i}(t) \leq \gamma, \quad t \in \mathbb{R}
$$

(b) For $i=1,2$, the function $l_{i}: \mathbb{R} \times \mathcal{O} \longrightarrow \mathbb{R}^{d}$ is continuous and bounded.

(A2) For $i=1,2$, there exists a complete orthonormal system $\left\{e_{i, k}\right\}_{k \in \mathbb{N}}$ in $\mathbb{M}$ and two sequences of nonnegative real numbers $\left\{\alpha_{i, k}\right\}_{k \in \mathbb{N}}$ and $\left\{\lambda_{i, k}\right\}_{k \in \mathbb{N}}$ such that

$$
\begin{aligned}
A_{i} e_{i, k} & =-\alpha_{i, k} e_{i, k}, \\
Q_{i} e_{i, k} & =\lambda_{i, k} e_{i, k}, \quad k \geq 1, \\
\kappa_{i} & :=\sum_{k=1}^{\infty} \lambda_{i, k}^{\rho_{i}}\left\|e_{i, k}\right\|_{\infty}^{2}<\infty, \\
\zeta_{i} & :=\sum_{k=1}^{\infty} \alpha_{i, k}^{-\beta_{i}}\left\|e_{i, k}\right\|_{\infty}^{2}<\infty,
\end{aligned}
$$

for some constants $\rho_{i} \in(2,+\infty]$ and $\beta_{i} \in(0,+\infty)$ such that

$$
\frac{\left[\beta_{i}\left(\rho_{i}-2\right)\right]}{\rho_{i}}<1 .
$$

Remark 1. For more comments and examples about the assumption (A2) of the operators $A_{i}$ and $Q_{i}$, the reader can read [12].

(A3) The mappings $b_{1}: \mathbb{R} \times \mathcal{O} \times \mathbb{R}^{2} \longrightarrow \mathbb{R}, f_{1}: \mathbb{R} \times \mathcal{O} \times$ $\mathbb{R} \longrightarrow \mathbb{R}, g_{1}: \mathbb{R} \times \mathcal{O} \times \mathbb{R} \times \mathbb{Z} \longrightarrow \mathbb{R}$ are measurable, and the mappings $b_{1}(t, \xi, \cdot): \mathbb{R}^{2} \longrightarrow \mathbb{R}$, $f_{1}(t, \xi, \cdot): \mathbb{R} \longrightarrow \mathbb{R}, \quad g_{1}(t, \xi, \cdot, z): \mathbb{R} \longrightarrow \mathbb{R}$ are Lipschitz continuous and linearly growing, uniformly with respect to $(t, \xi, z) \in \mathbb{R} \times \mathcal{O} \times \mathbb{Z}$. Moreover, for all $p \geq 1$, there exist positive constants $c_{1}$ and $c_{2}$, such that, for all $x_{1}, x_{2} \in \mathbb{R}$, we have

$$
\sup _{(t, \xi) \in \mathbb{R} \times \mathcal{O}} \int_{\mathbb{Z}}\left|g_{1}\left(t, \xi, x_{1}, z\right)\right|^{p} v_{1}(\mathrm{~d} z)
$$

$\leq c_{1}\left(1+\left|x_{1}\right|^{p}\right)$,

$$
\begin{aligned}
& \sup _{(t, \xi) \in \mathbb{R} \times \mathcal{O}} \int_{\mathbb{Z}}\left|g_{1}\left(t, \xi, x_{1}, z\right)-g_{1}\left(t, \xi, x_{2}, z\right)\right|^{p} v_{1}(\mathrm{~d} z) \\
& \leq c_{2}\left|x_{1}-x_{2}\right|^{p} .
\end{aligned}
$$

(A4) The mappings $b_{2}: \mathbb{R} \times \mathcal{O} \times \mathbb{R}^{2} \longrightarrow \mathbb{R}, f_{2}: \mathbb{R} \times \mathcal{O} \times$ $\mathbb{R}^{2} \longrightarrow \mathbb{R}, g_{2}: \mathbb{R} \times \mathcal{O} \times \mathbb{R}^{2} \times \mathbb{Z} \longrightarrow \mathbb{R}$ are measurable, and the mappings $b_{2}(t, \xi, \cdot): \mathbb{R}^{2} \longrightarrow \mathbb{R}$, $f_{2}(t, \xi, \cdot): \mathbb{R}^{2} \longrightarrow \mathbb{R}, g_{2}(t, \xi, \cdot, z): \mathbb{R}^{2} \longrightarrow \mathbb{R}$ are Lipschitz continuous and linearly growing, uniformly with respect to $(t, \xi, z) \in \mathbb{R} \times \mathcal{O} \times \mathbb{Z}$.
Moreover, for all $q \geq 1$, there exist positive constants $c_{3}$ and $c_{4}$, such that, for all $\left(x_{i}, y_{i}\right) \in \mathbb{R}^{2}, i=1,2$, we have

$$
\begin{aligned}
& \sup _{(t, \xi) \in \mathbb{R} \times \mathcal{O}} \int_{\mathbb{Z}}\left|g_{2}\left(t, \xi, x_{1}, y_{1}, z\right)\right|^{q} v_{2}(\mathrm{~d} z) \\
& \quad \leq c_{3}\left(1+\left|x_{1}\right|^{q}+\left|y_{1}\right|^{q}\right) \\
& \sup _{(t, \xi) \in \mathbb{R} \times \mathcal{O}} \int_{\mathbb{Z}} \mid g_{2}\left(t, \xi, x_{1}, y_{1}, z\right) \\
& \quad-\left.g_{2}\left(t, \xi, x_{2}, y_{2}, z\right)\right|^{q} v_{2}(\mathrm{~d} z) \\
& \leq c_{4}\left(\left|x_{1}-x_{2}\right|^{q}+\left|y_{1}-y_{2}\right|^{q}\right)
\end{aligned}
$$

Remark 2. For any $(t, \xi) \in \mathbb{R} \times \mathcal{O}$ and $x, y, h \in \mathbb{U}, z \in \mathbb{Z}$, we shall set

$$
\begin{aligned}
B_{1}(t, x, y)(\xi) & :=b_{1}(t, \xi, x(\xi), y(\xi)), \\
B_{2}(t, x, y)(\xi) & :=b_{2}(t, \xi, x(\xi), y(\xi)), \\
{\left[F_{1}(t, x) h\right](\xi) } & :=f_{1}(t, \xi, x(\xi)) h(\xi), \\
{\left[F_{2}(t, x, y) h\right](\xi) } & :=f_{2}(t, \xi, x(\xi), y(\xi)) h(\xi), \\
{\left[G_{1}(t, x, z) h\right](\xi) } & :=g_{1}(t, \xi, x(\xi), z) h(\xi), \\
{\left[G_{2}(t, x, y, z) h\right](\xi) } & :=g_{2}(t, \xi, x(\xi), y(\xi), z) h(\xi) .
\end{aligned}
$$

Due to $(\mathrm{A} 3)$ and $(\mathrm{A} 4)$, for any fixed $(t, z) \in(\mathbb{R}, \mathbb{Z})$, the mappings

$$
\begin{aligned}
& B_{1}(t, \cdot): \mathbb{W} \times \mathbb{W} \longrightarrow \mathbb{H}, \quad B_{2}(t, \cdot): \mathbb{H} \times \mathbb{W} \longrightarrow \mathbb{M}, \\
& F_{1}(t, \cdot): \mathbb{W} \longrightarrow \mathscr{L}(\mathbb{H}), \quad F_{2}(t, \cdot): \mathbb{H} \times \mathbb{W} \longrightarrow \mathscr{L}(\mathbb{H}), \\
& G_{1}(t, \cdot, z): \mathbb{W} \longrightarrow \mathscr{L}(\mathbb{W}), \quad G_{2}(t, \cdot, z): \mathbb{W} \times \mathbb{W} \longrightarrow \mathscr{L}(\mathbb{H}),
\end{aligned}
$$

are Lipschitz continuous and have linear growth conditions.

Now, for $i=1,2$, we define

$$
\gamma_{i}(t, s):=\int_{s}^{t} \gamma_{i}(r) \mathrm{d} r, \quad s<t,
$$

and for any $\epsilon>0$ and $\beta \geq 0$, set

$$
U_{\beta, \epsilon, i}(t, s)=e^{1 / \epsilon \gamma_{i}(t, s) A_{i}-\beta / \epsilon(t-s)}, \quad s<t .
$$

For $\epsilon=1$, we write $U_{\beta, i}(t, s)$, and for $\epsilon=1$ and $\beta=0$, we write $U_{i}(t, s)$.

Next, for any $\epsilon>0, \beta \geq 0$ and for any $u \in \mathscr{C}\left([s, t] ; W_{0}^{1, p}(\mathcal{O})\right)$, we define

$$
\psi_{\beta, \epsilon, i}(u ; s)(r)=\frac{1}{\epsilon} \int_{s}^{r} U_{\beta, \epsilon, i}(r, \rho) L_{i}(\rho) u(\rho) \mathrm{d} \rho, \quad s<r<t .
$$

In the case $\epsilon=1$, we write $\psi_{\beta, i}(u ; s)(r)$, and in the case $\epsilon=1$ and $\beta=0$, we write $\psi_{i}(u ; s)(r)$.

Remark 3. By using the same argument as Chapter 5 of the book [40], we can get that there exists a unique evolution system $U(t, s)=e^{A \int_{s}^{t} \gamma(r) \mathrm{d} r}(0 \leq s \leq t \leq T)$ for $\gamma(t) A$. Moreover, we also can get that $\psi_{\beta, \epsilon, i}(u ; s)(t)$ is the solution of 


$$
\mathrm{d} u(t)=\frac{1}{\epsilon}\left(A_{i}(t)-\beta\right) u(t) \mathrm{d} t, \quad t>s, u(s)=0 .
$$

\section{A Priori Bounds for the Solution}

With all notations introduced above, system (1) can be rewritten in the following abstract form:

$$
\begin{aligned}
\mathrm{d} u_{\epsilon}(t)= & {\left[A_{1}(t) u_{\epsilon}(t)+B_{1}\left(t, u_{\epsilon}(t), v_{\epsilon}(t)\right)\right] \mathrm{d} t } \\
& +F_{1}\left(t, u_{\epsilon}(t)\right) \mathrm{d} \omega^{Q_{1}}(t) \\
& +\int_{\mathbb{Z}} G_{1}\left(t, u_{\epsilon}(t), z\right) \widetilde{N}_{1}(\mathrm{~d} t, \mathrm{~d} z), \\
\mathrm{d} v_{\epsilon}(t)= & \frac{1}{\epsilon}\left[\left(A_{2}(t)-\alpha\right) v_{\epsilon}(t)+B_{2}\left(t, u_{\epsilon}(t), v_{\epsilon}(t)\right)\right] \mathrm{d} t \\
& +\frac{1}{\sqrt{\epsilon}} F_{2}\left(t, u_{\epsilon}(t), v_{\epsilon}(t)\right) \mathrm{d} \omega^{Q_{2}}(t) \\
& +\int_{\mathbb{Z}} G_{2}\left(t, u_{\epsilon}(t), v_{\epsilon}(t), z\right) \widetilde{N}_{2}^{\epsilon}(\mathrm{d} t, \mathrm{~d} z), \\
u_{\epsilon}(0)= & x, \quad v_{\epsilon}(0)=y .
\end{aligned}
$$

According to Remark 2, we know that the coefficients of system (28) satisfy global Lipschitz and linear growth conditions, and the assumptions (A1)-(A4) are uniform with respect to $t \in \mathbb{R}$. So, using the same argument as $[28,41,42]$, it is easy to prove that, for any $\epsilon>0, T>0$ and $x, y \in \mathbb{U}$, there exists a unique adapted mild solution $\left(u_{\epsilon}, v_{\epsilon}\right)$ to system $(28)$ in $L^{p}(\Omega ; D([0, T] ; \mathbb{W} \times \mathbb{U}))$. This means that there exist two unique adapted processes $u_{\epsilon}$ and $v_{\epsilon}$ in $L^{p}(\Omega ; D([0, T] ; \mathbb{H}))$ such that

$$
\left\{\begin{array}{l}
u_{\epsilon}(t)=U_{1}(t, 0) x+\psi_{1}\left(u_{\epsilon} ; 0\right)(t) \\
\quad+\int_{0}^{t} U_{1}(t, r) B_{1}\left(r, u_{\epsilon}(r), v_{\epsilon}(r)\right) \mathrm{d} r \\
\quad+\int_{0}^{t} U_{1}(t, r) F_{1}\left(r, u_{\epsilon}(r)\right) d w^{Q_{1}}(r) \\
\quad+\int_{0}^{t} \int_{\mathbb{Z}} U_{1}(t, r) G_{1}\left(r, u_{\epsilon}(r), z\right) \widetilde{N}_{1}(\mathrm{~d} r, \mathrm{~d} z), \\
\quad+\frac{1}{\epsilon} \int_{0}^{t} U_{\alpha, \epsilon, 2}(t, r) B_{2}\left(r, u_{\epsilon}(r), v_{\epsilon}(r)\right) \mathrm{d} r \\
\quad+\frac{1}{\sqrt{\epsilon}} \int_{0}^{t} U_{\alpha, \epsilon, 2}(t, r) F_{2}\left(r, u_{\epsilon}(r), v_{\epsilon}(r)\right) \mathrm{d} w^{Q_{2}}(r) \\
\quad+\int_{0}^{t} \int_{\mathbb{Z}} U_{\alpha, \epsilon, 2}(t, r) G_{2}\left(r, u_{\epsilon}(r), v_{\epsilon}(r), z\right) \widetilde{N}_{2}^{\epsilon}(\mathrm{d} r, \mathrm{~d} z) .
\end{array}\right.
$$

Lemma 1. Under (A1)-(A4), for any $p \geq 1$ and $T>0$, there exists a positive constant $c_{p, T}$, such that for any $x, y \in \mathbb{H}$ and $\epsilon \in(0,1]$, we have

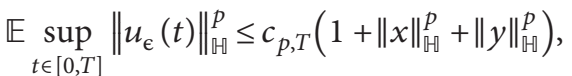

$$
\begin{aligned}
& \int_{0}^{T} \mathbb{E}\left\|v_{\epsilon}(t)\right\|_{\mathbb{H}}^{p} \mathrm{~d} t \leq c_{p, T}\left(1+\|x\|_{\mathbb{H}}^{p}+\|y\|_{\mathbb{H}}^{p}\right) .
\end{aligned}
$$

Proof. For fixed $\epsilon \in(0,1]$ and $x, y \in \mathbb{U}$, for any $t \in[0, T]$, we denote

$$
\begin{aligned}
& \Gamma_{1, \epsilon}(t):=\int_{0}^{t} U_{1}(t, r) F_{1}\left(r, u_{\epsilon}(r)\right) \mathrm{d} w^{Q_{1}}(r), \\
& \Psi_{1, \epsilon}(t):=\int_{0}^{t} \int_{\mathbb{Z}} U_{1}(t, r) G_{1}\left(r, u_{\epsilon}(r), z\right) \tilde{N}_{1}(\mathrm{~d} r, \mathrm{~d} z) .
\end{aligned}
$$

Setting $\Lambda_{1, \epsilon}(t):=u_{\epsilon}(t)-\Gamma_{1, \epsilon}(t)-\Psi_{1, \epsilon}(t)$, we have

$$
\begin{aligned}
\frac{\mathrm{d}}{\mathrm{d} t} \Lambda_{1, \epsilon}(t)= & \gamma_{1}(t) A_{1} \Lambda_{1, \epsilon}(t)+L_{1}(t)\left(\Lambda_{1, \epsilon}(t)+\Gamma_{1, \epsilon}(t)\right. \\
& \left.+\Psi_{1, \epsilon}(t)\right) B_{1}\left(t, \Lambda_{1, \epsilon}(t)+\Gamma_{1, \epsilon}(t)+\Psi_{1, \epsilon}(t), v_{\epsilon}(t)\right),
\end{aligned}
$$

$$
\Lambda_{1, \mathrm{\epsilon}}(0)=x \text {. }
$$

For any $p \geq 2$, because $B_{1}(\cdot)$ is Lipschitz continuous, using Young's inequality, we have

$$
\begin{aligned}
\frac{1}{p} \frac{\mathrm{d}}{\mathrm{d} t}\left\|\Lambda_{1, \epsilon}(t)\right\|_{\mathbb{H}}^{p}=\left\langle\gamma_{1}(t) A_{1} \Lambda_{1, \epsilon}(t), \Lambda_{1, \epsilon}(t)\right\rangle_{\mathbb{H}}\left\|\Lambda_{1, \epsilon}(t)\right\|_{\mathbb{H}}^{p-2} \\
\quad+\left\langle L_{1}(t)\left(\Lambda_{1, \epsilon}(t)+\Gamma_{1, \epsilon}(t)+\Psi_{1, \epsilon}(t)\right), \Lambda_{1, \epsilon}(t)\right\rangle_{\mathbb{H}}\left\|\Lambda_{1, \epsilon}(t)\right\|_{\mathbb{M}}^{p-2} \\
\quad+\left\langle B_{1}\left(t, \Lambda_{1, \epsilon}(t)+\Gamma_{1, \epsilon}(t)+\Psi_{1, \epsilon}(t), v_{\epsilon}(t)\right)\right. \\
\left.\quad-B_{1}\left(t, \Gamma_{1, \epsilon}(t)+\Psi_{1, \epsilon}(t), v_{\epsilon}(t)\right), \Lambda_{1, \epsilon}(t)\right\rangle_{\mathbb{H}}\left\|\Lambda_{1, \epsilon}(t)\right\|_{\mathbb{H}}^{p-2} \\
\quad+\left\langle B_{1}\left(t, \Gamma_{1, \epsilon}(t)+\Psi_{1, \epsilon}(t), v_{\epsilon}(t)\right)\right. \\
\left.\quad-B_{1}\left(t, \Psi_{1, \epsilon}(t), v_{\epsilon}(t)\right), \Lambda_{1, \epsilon}(t)\right\rangle_{\mathbb{H}}\left\|\Lambda_{1, \epsilon}(t)\right\|_{\mathbb{H}}^{p-2} \\
\quad+\left\langle B_{1}\left(t, \Psi_{1, \epsilon}(t), v_{\epsilon}(t)\right), \Lambda_{1, \epsilon}(t)\right\rangle_{\mathbb{H}}\left\|\Lambda_{1, \epsilon}(t)\right\|_{\mathbb{H}}^{p-2} \\
\leq c\left\|\Lambda_{1, \epsilon}(t)\right\|_{\mathbb{H}}^{p}+c\left\|\Gamma_{1, \epsilon}(t)\right\|_{\mathbb{H}}\left\|\Lambda_{1, \epsilon}(t)\right\|_{\mathbb{H}}^{p-1} \\
\quad+c\left\|\Psi_{1, \epsilon}(t)\right\|_{\mathbb{H}}\left\|\Lambda_{1, \epsilon}(t)\right\|_{\mathbb{H}}^{p-1} \\
\quad+c\left\|B_{1}\left(t, \Psi_{1, \epsilon}(t), v_{\epsilon}(t)\right)\right\|_{\mathbb{H}}\left\|\Lambda_{1, \epsilon}(t)\right\|_{\mathbb{H}}^{p-1} \\
\quad \leq c_{p}\left\|\Lambda_{1, \epsilon}(t)\right\|_{\mathbb{H}}^{p}+c_{p}\left(1+\left\|\Gamma_{1, \epsilon}(t)\right\|_{\mathbb{H}}^{p}+\left\|\Psi_{1, \epsilon}(t)\right\|_{\mathbb{H}}^{p}+\left\|v_{\epsilon}(t)\right\|_{\mathbb{H}}^{p}\right) .
\end{aligned}
$$

This implies that

$$
\begin{gathered}
\left\|\Lambda_{1, \epsilon}(t)\right\|_{\mathbb{G}}^{p} \leq e^{c_{p} t}\|x\|_{\mathbb{G}}^{p}+c_{p} \int_{0}^{t} e^{c_{p}(t-r)}\left(1+\left\|\Gamma_{1, \epsilon}(r)\right\|_{\mathbb{W}}^{p}\right. \\
\left.+\left\|\Psi_{1, \epsilon}(r)\right\|_{\mathbb{G}}^{p}+\left\|v_{\epsilon}(r)\right\|_{\mathbb{G}}^{p}\right) \mathrm{d} r .
\end{gathered}
$$


According to the definition of $\Lambda_{1, \epsilon}(t)$, for any $t \in[0, T]$, we have

$$
\begin{aligned}
\left\|u_{\epsilon}(t)\right\|_{\mathscr{H}}^{p} \leq & c_{p, T}\left(1+\|x\|_{\mathbb{H}}^{p}+\sup _{r \in[0, T]}\left\|\Gamma_{1, \epsilon}(r)\right\|_{\mathbb{H}}^{p}+\sup _{r \in[0, T]}\left\|\Psi_{1, \epsilon}(r)\right\|_{\mathbb{H}}^{p}\right) \\
& +c_{p, T} \int_{0}^{T}\left\|v_{\epsilon}(r)\right\|_{\mathscr{H}}^{p} \mathrm{~d} r .
\end{aligned}
$$

So,

$$
\begin{aligned}
& \mathbb{E} \sup _{t \in[0, T]}\left\|u_{\epsilon}(t)\right\|_{\mathbb{W}}^{p} \leq c_{p, T}\left(1+\|x\|_{\mathbb{W}}^{p}+\mathbb{E} \sup _{t \in[0, T]}\left\|\Gamma_{1, \epsilon}(t)\right\|_{\mathbb{H}}^{p}\right. \\
& \left.+\mathbb{E} \sup _{t \in[0, T]}\left\|\Psi_{1, \epsilon}(t)\right\|_{\mathbb{H}}^{p}\right)+c_{p, T} \int_{0}^{T} \mathbb{E}\left\|v_{\epsilon}(r)\right\|_{\mapsto \mathbb{T}}^{p} \mathrm{~d} r .
\end{aligned}
$$

According to Lemma 4.1 in [12] with $\theta=0$, it is easy to prove that

$$
\mathbb{E} \sup _{t \in[0, T]}\left\|\Gamma_{1, \mathcal{\epsilon}}(t)\right\|_{\mathbb{W}}^{p} \leq c_{p, T} \int_{0}^{T}\left(1+\mathbb{E}\left\|u_{\epsilon}(r)\right\|_{\mathbb{W}}^{p}\right) \mathrm{dr} .
$$

Due to (A3), using Kunita's first inequality, we get

$$
\begin{aligned}
& \mathbb{E} \sup _{t \in[0, T]}\left\|\Psi_{1, \epsilon}(t)\right\|_{\mathbb{H}}^{p} \leq c_{p} \mathbb{E}\left(\int_{0}^{T} \int_{\mathbb{Z}}\left\|e^{\gamma_{1}(t, r) A_{1}} G_{1}\left(r, u_{\epsilon}(r), z\right)\right\|_{\mathbb{H}}^{2} v_{1}(\mathrm{~d} z) \mathrm{d} r\right)^{p / 2}+c_{p} \mathbb{E} \int_{0}^{T} \int_{\mathbb{Z}}\left\|e^{\gamma_{1}(t, r) A_{1}} G_{1}\left(r, u_{\epsilon}(r), z\right)\right\|_{\mathbb{H}}^{p} v_{1}(\mathrm{~d} z) \mathrm{d} r \\
& \leq c_{p} \mathbb{E}\left(\int_{0}^{T}\left(1+\left\|u_{\epsilon}(r)\right\|_{\mathbb{H}}^{2}\right) \mathrm{d} r\right)^{p / 2}+c_{p} \mathbb{E} \int_{0}^{T}\left(1+\left\|u_{\epsilon}(r)\right\|_{\mathbb{H}}^{p}\right) \mathrm{d} r \leq c_{p, T} \int_{0}^{T}\left(1+\mathbb{E}\left\|u_{\epsilon}(r)\right\|_{\mathbb{H}}^{p}\right) \mathrm{d} r,
\end{aligned}
$$

$\mathbb{E} \sup _{t \in[0, T]}\left\|\Psi_{1, \mathcal{\epsilon}}(t)\right\|_{\mathbb{W}}^{p} \leq c_{p, T} \int_{0}^{T}\left(1+\mathbb{E}\left\|u_{\epsilon}(r)\right\|_{\mathbb{H}}^{p}\right) \mathrm{d} r$.

Substituting (38) and (39) into (37), we obtain

$$
\begin{aligned}
& \operatorname{Expp}_{t \in[0, T]}\left\|u_{\epsilon}(t)\right\|_{\mathbb{H}}^{p} \leq c_{p, T}\left(1+\|x\|_{\mathbb{H}}^{p}+\int_{0}^{T} \mathbb{E}\left\|v_{\epsilon}(r)\right\|_{\mathbb{H}}^{p} \mathrm{~d} r\right) \\
& +c_{p, T} \int_{0}^{T} \mathbb{E} \sup _{\sigma \in[0, r]}\left\|u_{\epsilon}(\sigma)\right\|_{\mathbb{H}}^{p} \mathrm{~d} r .
\end{aligned}
$$

Now, we have to estimate

$$
\int_{0}^{T} \mathbb{E}\left\|v_{\epsilon}(r)\right\|_{\bullet \rightarrow}^{p} \mathrm{~d} r .
$$

For any $t \in[0, T]$, we set

$$
\begin{aligned}
\Gamma_{2, \epsilon}(t) & :=\frac{1}{\sqrt{\epsilon}} \int_{0}^{t} U_{\alpha, \epsilon, 2}(t, r) F_{2}\left(r, u_{\epsilon}(r), v_{\epsilon}(r)\right) \mathrm{d} w^{Q_{2}}(r), \\
\Psi_{2, \epsilon}(t) & :=\int_{0}^{t} \int_{\mathbb{Z}} U_{\alpha, \epsilon, 2}(t, r) G_{2}\left(r, u_{\epsilon}(r), v_{\epsilon}(r), z\right) \widetilde{N}_{2}^{\epsilon}(\mathrm{d} r, \mathrm{~d} z) .
\end{aligned}
$$

Let $\Lambda_{2, \epsilon}(t):=v_{\epsilon}(t)-\Gamma_{2, \epsilon}(t)-\Psi_{2, \epsilon}(t)$, we have

$$
\frac{\mathrm{d}}{\mathrm{d} t} \Lambda_{2, \epsilon}(t)=\frac{1}{\epsilon}\left(\gamma_{2}(t) A_{2}-\alpha\right) \Lambda_{2, \epsilon}(t)+\frac{1}{\epsilon} L_{2}(t)
$$

$$
\begin{aligned}
& \cdot\left(\Lambda_{2, \epsilon}(t)+\Gamma_{2, \epsilon}(t)+\Psi_{2, \epsilon}(t)\right) \\
& +\frac{1}{\epsilon} B_{2}\left(t, u_{\epsilon}(t), \Lambda_{2, \epsilon}(t)+\Gamma_{2, \epsilon}(t)\right. \\
& \left.+\Psi_{2, \epsilon}(t)\right), \quad \Lambda_{2, \epsilon}(0)=y .
\end{aligned}
$$

For any $p \geq 2$, because $\alpha>0$ is large enough, by proceeding as in (34), we can get

$$
\begin{aligned}
\frac{1}{p} & \frac{\mathrm{d}}{\mathrm{d} t}\left\|\Lambda_{2, \epsilon}(t)\right\|_{\mathbb{M}}^{p} \leq-\frac{\alpha}{2 \epsilon}\left\|\Lambda_{2, \epsilon}(t)\right\|_{\mathbb{M}}^{p} \\
& +\frac{c_{p}}{\epsilon}\left(1+\left\|u_{\epsilon}(t)\right\|_{\mathbb{W}}^{p}+\left\|\Gamma_{2, \epsilon}(t)\right\|_{\mathbb{W}}^{p}+\left\|\Psi_{2, \epsilon}(t)\right\|_{\mathbb{M}}^{p}\right) .
\end{aligned}
$$

According to the Gronwall inequality, we have

$\left\|\Lambda_{2, \epsilon}(t)\right\|_{[\rightarrow}^{p} \leq \frac{c_{p}}{\epsilon} \int_{0}^{t} e^{-(\alpha p / 2 \epsilon)(t-r)}$

$$
\cdot\left(1+\left\|u_{\mathcal{\epsilon}}(r)\right\|_{\mathbb{H}}^{p}+\left\|\Gamma_{2, \mathrm{\epsilon}}(r)\right\|_{\mathbb{H}}^{p}+\left\|\Psi_{2, \mathrm{\epsilon}}(r)\right\|_{\mathbb{H}}^{p}\right) \mathrm{d} r+e^{-(\alpha p / 2 \mathrm{e}) t}\|y\|_{\mathbb{H}}^{p} .
$$


According to the definition of $\Lambda_{2, \epsilon}(t)$, for any $t \in[0, T]$, we have

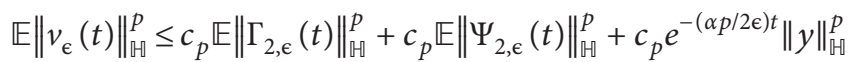

$$
\begin{aligned}
& +\frac{c_{p}}{\epsilon} \int_{0}^{t} e^{-(\alpha p / 2 \epsilon)(t-r)}\left(1+\mathbb{E}\left\|u_{\epsilon}(r)\right\|_{\mathbb{G}}^{p}+\mathbb{E}\left\|\Gamma_{2, \epsilon}(r)\right\|_{\mathbb{H}}^{p}\right. \\
& \left.+\mathbb{E}\left\|\Psi_{2, \mathrm{\epsilon}}(r)\right\|_{\mathbb{H}}^{p}\right) \mathrm{d} r
\end{aligned}
$$

Therefore, by integrating with respect to $t$, using Young's inequality, we obtain

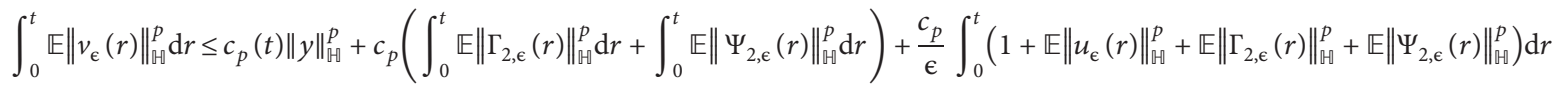

$$
\begin{aligned}
& \times \int_{0}^{t} e^{-(\alpha p / 2 \mathrm{e}) r} \mathrm{~d} r \leq c_{p}(t)\left(1+\|y\|_{H \in H}^{p}\right)+c_{p}\left(\int_{0}^{t} \mathbb{E}\left\|u_{\mathrm{e}}(r)\right\|_{H}^{p} \mathrm{~d} r+\int_{0}^{t} \mathbb{E}\left\|\Gamma_{2, \mathrm{e}}(r)\right\|_{H \in H}^{p} \mathrm{~d} r+\int_{0}^{t} \mathbb{E}\left\|\Psi_{2, \mathrm{e}}(r)\right\|_{H}^{p} \mathrm{~d} r\right) .
\end{aligned}
$$

According to the Burkholder-Davis-Gundy inequality, by proceeding as Proposition 4.2 in [12], we can easily get

$$
\int_{0}^{t} \mathbb{E}\left\|\Gamma_{2, \epsilon}(r)\right\|_{\mathbb{Q}}^{p} \mathrm{~d} r \leq c_{p}(t) \int_{0}^{t}\left(1+\mathbb{E}\left\|u_{\epsilon}(r)\right\|_{\mathbb{Q}}^{p}+\mathbb{E}\left\|v_{\epsilon}(r)\right\|_{\mathbb{H}}^{p}\right) \mathrm{d} r .
$$

Concerning the stochastic term $\Psi_{2, \epsilon}(t)$, using Kunita's first inequality, we have

$$
\begin{aligned}
\mathbb{E}\left\|\Psi_{2, \epsilon}(t)\right\|_{\mathbb{H}}^{p} \leq & c_{p} \mathbb{E}\left(\frac{1}{\epsilon} \int_{0}^{t} \int_{\mathbb{Z}}\left\|e^{-(\alpha / \epsilon)(t-r)} e^{\left(\gamma_{2}(t, r) / \epsilon\right) A_{2}} G_{2}\left(r, u_{\epsilon}(r), v_{\epsilon}(r), z\right)\right\|_{\mathbb{H}}^{2} v_{2}(\mathrm{~d} z) \mathrm{d} r\right)^{p / 2} \\
& +\frac{c_{p}}{\epsilon} \mathbb{E} \int_{0}^{t} \int_{\mathbb{Z}}\left\|e^{-(\alpha / \epsilon)(t-r)} e^{\left(\gamma_{2}(t, r) / \epsilon\right) A_{2}} G_{2}\left(r, u_{\epsilon}(r), v_{\epsilon}(r), z\right)\right\|_{\mathbb{H}}^{p} v_{2}(\mathrm{~d} z) \mathrm{d} r \\
\leq & \frac{c_{p}}{\epsilon} \mathbb{E} \int_{0}^{t} e^{-(\alpha(p-2) \epsilon)(t-r)}\left(1+\left\|u_{\epsilon}(r)\right\|_{\mathbb{H}}^{p}+\left\|v_{\epsilon}(r)\right\|_{\mathbb{H}}^{p}\right) \mathrm{d} r\left(\int_{0}^{t} e^{-(\alpha p / \epsilon)(p-2)(t-r)} \mathrm{d} r\right)^{(p-2) / 2} \\
& +\frac{c_{p}}{\epsilon} \mathbb{E} \int_{0}^{t} e^{-(\alpha p / \epsilon)(t-r)}\left(1+\left\|u_{\epsilon}(r)\right\|_{\mathbb{H}}^{p}+\left\|v_{\epsilon}(r)\right\|_{\mathbb{H}}^{p}\right) \mathrm{d} r \leq \frac{c_{p}}{\epsilon} \int_{0}^{t} e^{-(\alpha p / 2 \epsilon)(t-r)}\left(1+\mathbb{E}\left\|u_{\epsilon}(r)\right\|_{\mathbb{H}}^{p}+\mathbb{E}\left\|v_{\epsilon}(r)\right\|_{\mathbb{H}}^{p}\right) \mathrm{d} r .
\end{aligned}
$$

By integrating with respect to $t$ both sides and using Young's inequality, we have

$$
\begin{aligned}
& \int_{0}^{t} \mathbb{E}\left\|\Psi_{2, \epsilon}(r)\right\|_{\mathscr{H}}^{p} \mathrm{~d} r \leq \frac{c_{p}}{\epsilon} \int_{0}^{t} e^{-(\alpha p / 2 \epsilon) r} \mathrm{~d} r \cdot \int_{0}^{t}\left(1+\mathbb{E}\left\|u_{\epsilon}(r)\right\|_{H}^{p}\right. \\
& \left.+\mathbb{E}\left\|v_{\epsilon}(r)\right\|_{H}^{p}\right) \mathrm{d} r \leq c_{p}(t) \int_{0}^{t}\left(1+\mathbb{E}\left\|u_{\mathcal{\epsilon}}(r)\right\|_{\mathscr{H}}^{p}+\mathbb{E}\left\|v_{\epsilon}(r)\right\|_{H}^{p}\right) \mathrm{d} r .
\end{aligned}
$$

Substituting (49) and (51) into (48), we get

$$
\begin{aligned}
& \int_{0}^{t} \mathbb{E}\left\|v_{\epsilon}(r)\right\|_{\mathbb{W}}^{p} \mathrm{~d} r \leq c_{p}(t)\left(1+\|y\|_{\mathbb{M}}^{p}+\int_{0}^{t} \mathbb{E}\left\|u_{\epsilon}(r)\right\|_{\mathbb{W}}^{p} \mathrm{~d} r\right) \\
& \quad+c_{p, T}(t) \int_{0}^{t} \mathbb{E}\left\|v_{\epsilon}(r)\right\|_{\mathbb{W}}^{p} \mathrm{~d} r .
\end{aligned}
$$

As $c_{p}(0)=0$ and $c_{p}(t)$ is a continuous increasing function, we can fix $t_{0}>0$, such that for any $t \leq t_{0}$, we have $c_{p}(t) \leq 1 / 2$; so, 


$$
\int_{0}^{t} \mathbb{E}\left\|v_{\epsilon}(r)\right\|_{\mathbb{H}}^{p} \mathrm{~d} r \leq c_{p}(t)\left(1+\|y\|_{\mathbb{Q}}^{p}+\mathbb{E} \sup _{r \in[0, t]}\left\|u_{\epsilon}(r)\right\|_{\mathbb{H}}^{p}\right), \quad t \in\left[0, t_{0}\right] .
$$

Due to (41) and (53), for any $t \in\left[0, t_{0}\right]$, we can get

$$
\begin{aligned}
& \mathbb{E} \sup _{r \in[0, t]}\left\|u_{\epsilon}(r)\right\|_{\mathbb{G}}^{p} \leq c_{p, T}\left(1+\|x\|_{\mathbb{Q}}^{p}+\|y\|_{\mathbb{E}}^{p}\right) \\
& \quad+c_{p, T}(t) \mathbb{E} \sup _{r \in[0, t]}\left\|u_{\epsilon}(r)\right\|_{\mathbb{G}}^{p}+c_{p, T} \int_{0}^{t} \mathbb{E} \sup _{\sigma \in[0, r]}\left\|u_{\epsilon}(\sigma)\right\|_{\mathbb{G}}^{p} \mathrm{~d} r .
\end{aligned}
$$

Similarly, we also can fix $0<t_{1} \leq t_{0}$, such that for any $t \leq t_{1}$, we have $c_{p, T}(t) \leq 1 / 2$; so,

$$
\begin{aligned}
& \mathbb{E} \sup _{r \in[0, t]}\left\|u_{\epsilon}(r)\right\|_{\mathbb{G}}^{p} \leq c_{p, T}\left(1+\|x\|_{\mathbb{G}}^{p}+\|y\|_{\mathbb{G}}^{p}\right) \\
& \quad+c_{p, T} \int_{0}^{t} \mathbb{E} \sup _{\sigma \in[0, r]}\left\|u_{\epsilon}(\sigma)\right\|_{\mathbb{G}}^{p} \mathrm{~d} r, \quad t \in\left[0, t_{1}\right] .
\end{aligned}
$$

According to the Gronwall inequality, we get

$\mathbb{E} \sup _{r \in[0, t]}\left\|u_{\epsilon}(r)\right\|_{\mathbb{G}}^{p} \leq c_{p, T}\left(1+\|x\|_{\mathbb{Q}}^{p}+\|y\|_{\mathbb{\square}}^{p}\right), \quad t \in\left[0, t_{1}\right]$.

Substituting (56) into (53), it yields

$$
\int_{0}^{t} \mathbb{E}\left\|v_{\epsilon}(r)\right\|_{\mathbb{Q}}^{p} \mathrm{~d} r \leq c_{p, T}\left(1+\|x\|_{\mathbb{Q}}^{p}+\|y\|_{\mathbb{Q}}^{p}\right), \quad t \in\left[0, t_{1}\right] .
$$

For any $p \geq 2$, by repeating this in the intervals $\left[t_{1}, 2 t_{1}\right],\left[2 t_{1}, 3 t_{1}\right]$ etc., we can easily get (31). Substituting (31) into (41) and using the Gronwall inequality again, we get (30). Using the Hölder inequality, we can estimate (30) and (31) for $p=1$.

Lemma 2. Under (A1)-(A4), there exists $\bar{\theta}>0$, such that for any $T>0, p \geq 1, x \in \mathscr{D}\left(\left(-A_{1}\right)^{\theta}\right)$ with $\theta \in[0, \bar{\theta})$ and $y \in \mathbb{U}$, there exist a positive constant $c_{p, \theta, T}>0$ such that

$$
\sup _{\epsilon \in(0,1]} \mathbb{E} \sup _{t \in[0, T]}\left\|u_{\epsilon}(t)\right\|_{\theta}^{p} \leq c_{p, \theta, T}\left(1+\|x\|_{\theta}^{p}+\|y\|_{\mathbb{⿴ 囗 十}}^{p}\right) .
$$

Proof. Assuming that $x \in \mathscr{D}\left(\left(-A_{1}\right)^{\theta}\right)(\theta \geq 0)$, for any $t \in[0, T]$, we have

$$
\begin{aligned}
u_{\epsilon}(t)= & U_{1}(t, 0) x+\psi_{1}\left(u_{\epsilon} ; 0\right)(t) \\
& +\int_{0}^{t} U_{1}(t, r) B_{1}\left(r, u_{\epsilon}(r), v_{\epsilon}(r)\right) \mathrm{d} r \\
& +\int_{0}^{t} U_{1}(t, r) F_{1}\left(r, u_{\epsilon}(r)\right) \mathrm{d} w^{Q_{1}}(r) \\
& +\int_{0}^{t} \int_{\mathbb{Z}} U_{1}(t, r) G_{1}\left(r, u_{\epsilon}(r), z\right) \tilde{N}_{1}(\mathrm{~d} r, \mathrm{~d} z) .
\end{aligned}
$$

Concerning the second term $\psi_{1}\left(u_{\epsilon} ; 0\right)(t)$, we get

$$
\begin{aligned}
\left\|\psi_{1}\left(u_{\epsilon} ; 0\right)(t)\right\|_{\theta}^{p} & \leq c_{p}\left\|\int_{0}^{t}\left(-A_{1}\right)^{\theta} e^{\gamma_{1}(t, r) A_{1}} L_{1}(r) u_{\epsilon}(r) \mathrm{d} r\right\|_{\mathbb{T}}^{p} \\
& \leq c_{p, \theta}\left(\int_{0}^{t}(t-r)^{-\theta}\left\|L_{1}(r) u_{\epsilon}(r)\right\|_{\mathbb{Q}} \mathrm{d} r\right)^{p} \\
& \leq c_{p, \theta} \sup _{r \in[0, T]}\left\|u_{\epsilon}(r)\right\|_{\mathbb{G}}^{p}\left(\int_{0}^{t}(t-r)^{-\theta} \mathrm{d} r\right)^{p} \\
& \leq c_{p, \theta, T}\left(1+\|x\|_{\mathbb{Q}}^{p}+\|y\|_{\mathbb{Q}}^{p}\right) .
\end{aligned}
$$

For any $p \geq 2$, according to the proof of Proposition 4.3 in [12], and thanks to (30) and (31), it is possible to show that there exists a $\widetilde{\theta} \geq 0$, such that for any $\theta \leq \widetilde{\theta} \wedge 1 / 2$, we have

$$
\mathbb{E} \sup _{t \in[0, T]}\left\|\int_{0}^{t} U_{1}(t, r) B_{1}\left(r, u_{\epsilon}(r), v_{\epsilon}(r)\right) \mathrm{d} r\right\|_{\theta}^{p} \leq c_{p, \theta, T}\left(1+\|x\|_{\mathbb{H}}^{p}+\|y\|_{\mathbb{H}}^{p}\right) .
$$

$$
\mathbb{E} \sup _{t \in[0, T]}\left\|\Gamma_{1, \epsilon}(t)\right\|_{\theta}^{p} \leq c_{p, \theta, T}\left(1+\|x\|_{\mathbb{Q}}^{p}+\|y\|_{\mathbb{Q}}^{p}\right) .
$$

Concerning the stochastic term $\Psi_{1, \epsilon}(t)$, using the factorization argument, we have

$$
\Psi_{1, \epsilon}(t)=c_{\theta} \int_{0}^{t}(t-r)^{\theta-1} e^{\gamma_{1}(t, r) A_{1}} \phi_{\epsilon, \theta}(r) \mathrm{d} r
$$

where

$$
\phi_{\epsilon, \theta}(r)=\int_{0}^{r} \int_{\mathbb{Z}}(r-\sigma)^{-\theta} e^{\gamma_{1}(r, \sigma) A_{1}} G_{1}\left(\sigma, u_{\epsilon}(\sigma), z\right) \tilde{N}_{1}(\mathrm{~d} \sigma, \mathrm{d} z) .
$$

Next, for any $p \geq 2$, let $\widehat{\theta}=((1 / 4)-(1 / 2 p)) \wedge 1 / 2 p$, for any $\theta \leq \widehat{\theta}$, according to (A3) and Lemma 1 , using Kunita's first inequality and the Hölder inequality, we get 


$$
\begin{aligned}
\left\|\Psi_{1, \epsilon}(t)\right\|_{\theta}^{p} \leq & c_{\theta}\left(\int_{0}^{t}(t-r)^{\theta-1}\left\|\phi_{\epsilon, \theta}(r)\right\|_{\theta} \mathrm{d} r\right)^{p} \leq c_{\theta} \sup _{r \in[0, t]}\left\|\phi_{\epsilon, \theta}(r)\right\|_{\theta}^{p}\left(\int_{0}^{t}(t-r)^{\theta-1} \mathrm{~d} r\right)^{p} \\
\leq & c_{p, \theta, T} \sup _{r \in[0, t]}\left\|\int_{0}^{r} \int_{\mathbb{Z}}(r-\sigma)^{-\theta}\left(-A_{1}\right)^{\theta} e^{\gamma_{1}(r, \sigma) A_{1}} G_{1}\left(\sigma, u_{\epsilon}(\sigma), z\right) \widetilde{N}_{1}(\mathrm{~d} \sigma, \mathrm{d} z)\right\|_{\mathbb{B}}^{p} \\
\leq & c_{p, \theta, T} \sup _{r \in[0, t]}\left(\int_{0}^{r} \int_{\mathbb{Z}}(r-\sigma)^{-2 \theta}(r-\sigma)^{-2 \theta}\left\|G_{1}\left(\sigma, u_{\epsilon}(\sigma), z\right)\right\|_{\mathbb{G}}^{2} v_{1}(\mathrm{~d} z) \mathrm{d} \sigma\right)^{p / 2} \\
& +c_{p, \theta, T} \sup _{r \in[0, t]} \int_{0}^{r} \int_{\mathbb{Z}}(r-\sigma)^{-p \theta}(r-\sigma)^{-p \theta}\left\|G_{1}\left(\sigma, u_{\epsilon}(\sigma), z\right)\right\|_{\mathbb{H}}^{p} v_{1}(\mathrm{~d} z) \mathrm{d} \sigma \leq c_{p, \theta, T} \sup _{r \in[0, t]}\left[\int_{0}^{r}\left(\int_{\mathbb{Z}}\left\|G_{1}\left(\sigma, u_{\epsilon}(\sigma), z\right)\right\|_{\mathbb{B}}^{2} v_{1}(\mathrm{~d} z)\right)^{p / 2} \mathrm{~d} \sigma\right. \\
& \left.\times\left(\int_{0}^{r}(r-\sigma)^{-4 p \theta /(p-2)} \mathrm{d} \sigma\right)^{(p-2) / 2}\right]+c_{p, \theta, T} \sup _{r \in[0, t]}\left[\int_{0}^{r}(r-\sigma)^{-2 p \theta} \mathrm{d} \sigma \cdot\left(\sup _{\sigma \in[0, r]} \int_{\mathbb{Z}}\left\|G_{1}\left(\sigma, u_{\epsilon}(\sigma), z\right)\right\|_{\mathbb{B}}^{p} v_{1}(\mathrm{~d} z)\right)\right] \\
\leq & c_{p, \theta, T}\left(1+\sup _{\sigma \in[0, T]}\left\|u_{\epsilon}(\sigma)\right\|_{\mathbb{H}}^{p}\right) .
\end{aligned}
$$

So, due to (30), we get

$$
\mathbb{E} \sup _{t \in[0, T]}\left\|\Psi_{1, \epsilon}(t)\right\|_{\theta}^{p} \leq c_{p, \theta, T}\left(1+\|x\|_{\mathbb{Q}}^{p}+\|y\|_{\mathbb{Q}}^{p}\right) .
$$

Hence, if we choose $\bar{\theta}:=1 / 8 \wedge \tilde{\theta} \wedge \widehat{\theta}$, thanks to (60)-(62) and (66), for any $p \geq 2$ and $\theta<\bar{\theta}$, we get

$$
\mathbb{E} \sup _{t \in[0, T]}\left\|u_{\epsilon}(t)\right\|_{\theta}^{p} \leq c_{p, \theta, T}\left(1+\|x\|_{\theta}^{p}+\|y\|_{\mathbb{⿴}}^{p}\right) .
$$
$p=1$.
Using the Hölder inequality, we can estimate (58) for
Lemma 3. Under (A1)-(A4), for any $\theta \in[0, \bar{\theta})$ and $0 \leq h \leq 1$, there exists $\beta(\theta)>0$, such that, for any $T>0, p \geq 1, x \in \mathscr{D}\left(\left(-A_{1}\right)^{\theta}\right), y \in \mathbb{H}$, and $t \in[0, T]$, it holds

$$
\begin{aligned}
& \sup _{\epsilon \in(0,1]} \mathbb{E}\left\|u_{\epsilon}(t)-u_{\epsilon}(t+h)\right\|_{\mathbb{Q}}^{p} \\
& \quad \leq c_{p, \theta, T}\left(h^{\beta(\theta) p}+h\right)\left(1+\|x\|_{\theta}^{p}+\|y\|_{\mathbb{Q}}^{p}\right) .
\end{aligned}
$$

Proof. For any $t \geq 0$ with $t, t+h \in[0, T]$, we have

$$
\begin{aligned}
u_{\epsilon}(t+h)-u_{\epsilon}(t)= & \left(U_{1}(t+h, t)-I\right) u_{\epsilon}(t)+\psi_{1}\left(u_{\epsilon} ; t\right)(t+h)+\int_{t}^{t+h} U_{1}(t+h, r) B_{1}\left(r, u_{\epsilon}(r), v_{\epsilon}(r)\right) \mathrm{d} r \\
& +\int_{t}^{t+h} U_{1}(t+h, r) F_{1}\left(r, u_{\epsilon}(r)\right) \mathrm{d} w^{Q_{1}}(r) \\
& +\int_{t}^{t+h} \int_{\mathbb{Z}} U_{1}(t+h, r) G_{1}\left(r, u_{\epsilon}(r), z\right) \tilde{N}_{1}(\mathrm{~d} r, \mathrm{~d} z):=\sum_{i=1}^{5} \mathscr{I}_{t}^{i} .
\end{aligned}
$$

By proceeding as the proof of Proposition 4.4 in [12] and (60), fix $\theta \in[0, \bar{\theta})$, for any $p \geq 1$, and it is possible to show that

$$
\begin{aligned}
& \mathbb{E}\left\|\mathscr{I}_{t}^{1}\right\|_{\mathbb{Q}}^{p} \leq c_{p, \theta, T} h^{\theta p}\left(1+\|x\|_{\theta}^{p}+\|y\|_{\mathbb{Q}}^{p}\right), \\
& \mathbb{E}\left\|\mathscr{J}_{t}^{2}\right\|_{\mathbb{Q}}^{p} \leq c_{p, T} h^{p-1}\left(1+\|x\|_{\theta}^{p}+\|y\|_{\mathbb{Q}}^{p}\right),
\end{aligned}
$$

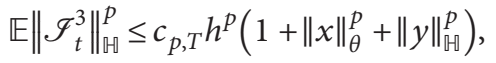

$$
\begin{aligned}
& \mathbb{E}\left\|\mathscr{I}_{t}^{4}\right\|_{\mathbb{Q}}^{p} \leq c_{p, T} h^{(p-2) / 2-\left(\beta_{1}\left(\rho_{1}-2\right) / \rho_{1}\right)(p / 2)}\left(1+\|x\|_{\theta}^{p}+\|y\|_{\mathbb{Q}}^{p}\right) .
\end{aligned}
$$

According to the proof of (39), using the Hölder inequality and (30), we have

$$
\begin{aligned}
& \mathbb{E}\left\|\mathscr{J}_{t}^{5}\right\|_{\mathbb{Q} \mathbb{T}}^{p} \leq c_{p} \mathbb{E}\left(\int_{t}^{t+h}\left(1+\left\|u_{\epsilon}(r)\right\|_{\mathbb{Q}}^{2}\right) \mathrm{d} r\right)^{p / 2} \\
& +c_{p} \mathbb{E} \int_{t}^{t+h}\left(1+\left\|u_{\epsilon}(r)\right\|_{\mathbb{G}}^{p}\right) \mathrm{d} r, \leq c_{p}\left(h^{(p-2) / 2}+1\right) \\
& \cdot \int_{t}^{t+h}\left(1+\mathbb{E}\left\|u_{\epsilon}(r)\right\|_{\mathbb{R}}^{p}\right) \mathrm{d} r \\
& \leq c_{p, T}\left(h^{\frac{p}{2}}+h\right)\left(1+\|x\|_{\mathbb{\square}}^{p}+\|y\|_{\mathbb{\square}}^{p}\right) .
\end{aligned}
$$

Then, if we take $\bar{p}>1$, such that

$$
\frac{\beta_{1}\left(\rho_{1}-2\right)}{\rho_{1}} \frac{\bar{p}}{\bar{p}-2}<1 \text {. }
$$

We can get 


$$
\begin{aligned}
& \mathbb{E}\left\|u_{\epsilon}(t+h)-u_{\epsilon}(t)\right\|_{\leftrightarrow}^{p} \\
& \leq c_{p, T}\left(h^{(1-1 / \bar{p}) p}+h^{p}+h^{\left((1 / 2)-(1 / \bar{p})-\beta_{1}\left(\rho_{1}-2\right) / 2 \rho_{1}\right) p}+h^{p / 2}+h\right) \\
& \times\left(1+\|x\|_{\mathbb{H}}^{p}+\|y\|_{\mathbb{H}}^{p}\right)+c_{p, \theta, T} h^{\theta p}\left(1+\|x\|_{\theta}^{p}+\|y\|_{\mathbb{H}}^{p}\right) .
\end{aligned}
$$

As we are assuming $|h| \leq 1$, (68) follows for any $p \geq \bar{p}$ by taking

$$
\beta(\theta):=\min \left\{\theta, 1-\frac{1}{\bar{p}}, 1, \frac{1}{2}-\frac{1}{\bar{p}}-\frac{\beta_{1}\left(\rho_{1}-2\right)}{2 \rho_{1}}, \frac{1}{2}\right\} .
$$

From the Hölder inequality, we can estimate (68) for $p<\bar{p}$

$$
\mathbb{E}\left\|u_{\epsilon}(t+h)-u_{\epsilon}(t)\right\|_{\mathbb{G}}^{p} \leq\left[\mathbb{E}\left\|u_{\epsilon}(t+h)-u_{\epsilon}(t)\right\|_{\mathbb{E}}^{\bar{p}}\right]^{p / \bar{p}} .
$$

So, we have (68).

According to the above lemma, we get that, for every $\epsilon \in(0,1]$, the function $u_{\epsilon}(t)$ is uniformly bounded about $t \in[0, T]$, and it is also equicontinuous at every point of $t \in[0, T]$. In view of Theorem 12.3 in [43], we can infer that the set $\left\{u_{\epsilon}\right\}_{\epsilon \in(0,1]}$ is relatively compact in $D([0, T] ; \mathbb{H})$. In addition, according to Theorem 13.2 in [43] and the above lemma, by using Chebyshev's inequality, we also can get that the family of probability measures $\left\{\mathscr{L}\left(u_{\epsilon}\right)\right\}_{\epsilon \in(0,1]}$ is tight in $\mathscr{P}(D([0, T] ; \nVdash))$.

\section{An Evolution Family of Measures for the Fast Equation}

For any frozen slow component $x \in \mathbb{H}$, any initial condition $y \in \mathbb{H}$, and any $s \in \mathbb{R}$, we introduce the following problem:

$$
\begin{aligned}
\mathrm{d} v(t)= & {\left[\left(A_{2}(t)-\alpha\right) v(t)+B_{2}(t, x, v(t))\right] \mathrm{d} t+F_{2}(t, x, v(t)) \mathrm{d} \bar{\omega}^{Q_{2}}(t) } \\
& +\int_{\mathbb{Z}} G_{2}(t, x, v(t), z) \tilde{N}_{2,}(\mathrm{~d} t, \mathrm{~d} z), \quad v(s)=y,
\end{aligned}
$$

where

$$
\begin{gathered}
\bar{w}^{Q_{2}}(t)= \begin{cases}w_{1}^{Q_{2}}(t), & \text { if } t \geq 0, \\
w_{2}^{Q_{2}}(-t), & \text { if } t<0,\end{cases} \\
\tilde{N}_{2^{\prime}}(t, z)= \begin{cases}\tilde{N}_{1,}(t, z), & \text { if } t \geq 0, \\
\tilde{N}_{3,}(-t, z), & \text { if } t<0,\end{cases}
\end{gathered}
$$

for two independent $Q_{2}$-Wiener processes $w_{1}^{Q_{2}}(t)$ and $w_{2}^{Q_{2}}(t)$ and two independent compensated Poisson measures $\tilde{N}_{1^{\prime}}(\mathrm{d} t, \mathrm{~d} z)$ and $\tilde{N}_{3^{\prime}}(\mathrm{d} t, \mathrm{~d} z)$ with the same Lévy measure are both defined as in Section 2.

According to the definition of the operator $\psi_{\alpha, 2}(\cdot ; s)$, we know that the mapping $\psi_{\alpha, 2}(\cdot ; s): \mathscr{C}([s, T] ; \mathbb{H}) \longrightarrow$ $\mathscr{C}([s, T] ; \mathbb{H})$ is a linearly bounded operator, and it is Lipschitz continuous. Hence, we have that, for any $x, y \in \mathbb{H}, p \geq 1$ and $s<T$, there exists a unique mild solution $v^{x}(\cdot ; s, y) \in L^{p}(\Omega ; D([0, T] ; \mathbb{W}))$ (see $\left.[28]\right)$ in the following form:

$$
\begin{aligned}
v^{x}(t ; s, y)= & U_{\alpha, 2}(t, s) y+\psi_{\alpha, 2}\left(v^{x}(\cdot ; s, y) ; s\right)(t) \\
& +\int_{s}^{t} U_{\alpha, 2}(t, r) B_{2}\left(r, x, v^{x}(r ; s, y)\right) \mathrm{d} r \\
& +\int_{s}^{t} U_{\alpha, 2}(t, r) F_{2}\left(r, x, v^{x}(r ; s, y)\right) \mathrm{d} \bar{w}^{Q_{2}}(r) \\
& +\int_{s}^{t} \int_{\mathbb{Z}} U_{\alpha, 2}(t, r) G_{2}\left(r, x, v^{x}(r ; s, y), z\right) \widetilde{N}_{2^{\prime}}(\mathrm{d} r, \mathrm{~d} z) .
\end{aligned}
$$

Moreover, if the space $D(\mathbb{R} ; \mathbb{U})$ endowed with the topology of uniform convergence on bounded intervals, an $\left\{\mathscr{F}_{t}\right\}_{t \in \mathbb{R}^{-}}$adapted process $v^{x} \in L^{p}(\Omega ; D([0, T] ; \mathbb{H}))$ is a mild solution of the equation:

$$
\begin{aligned}
\mathrm{d} v(t)= & {\left[\left(A_{2}(t)-\alpha\right) v(t)+B_{2}(t, x, v(t))\right] \mathrm{d} t } \\
& +F_{2}(t, x, v(t)) \mathrm{d} \bar{\omega}^{Q_{2}}(t) \\
& +\int_{\mathbb{Z}} G_{2}(t, x, v(t), z) \tilde{N}_{2}(\mathrm{~d} t, \mathrm{~d} z),
\end{aligned}
$$

where $t \in \mathbb{R}$. Then, for every $s<t$, we have

$$
\begin{aligned}
v^{x}(t)= & U_{\alpha, 2}(t, s) v^{x}(s)+\psi_{\alpha, 2}\left(v^{x} ; s\right)(t) \\
& +\int_{s}^{t} U_{\alpha, 2}(t, r) B_{2}\left(r, x, v^{x}(r)\right) \mathrm{d} r \\
& +\int_{s}^{t} U_{\alpha, 2}(t, r) F_{2}\left(r, x, v^{x}(r)\right) \mathrm{d} \bar{w}^{Q_{2}}(r) \\
& +\int_{s}^{t} \int_{\mathbb{Z}} U_{\alpha, 2}(t, r) G_{2}\left(r, x, v^{x}(r), z\right) \tilde{N}_{2,}(\mathrm{~d} r, \mathrm{~d} z) .
\end{aligned}
$$

In the following, for any $x \in \mathbb{U}$ and any adapted process $v$, we set

$$
\begin{aligned}
\Gamma_{\alpha}(v ; s)(t) & :=\int_{s}^{t} U_{\alpha, 2}(t, r) F_{2}(r, x, v(r)) \mathrm{d} \bar{w}^{Q_{2}}(r), \quad t>s, \\
\Psi_{\alpha}(v ; s)(t): & =\int_{s}^{t} \int_{\mathbb{Z}} U_{\alpha, 2}(t, r) G_{2}(r, x, v(r), z) \widetilde{N}_{2,}(\mathrm{~d} r, \mathrm{~d} z), \quad t>s .
\end{aligned}
$$

For any $0<\delta<\alpha$ and any $v_{1}$ and $v_{2}$ with $s<t$, by proceeding as in the proof of Lemma 7.1 in [44], it is possible to show that there exists $\bar{p}>1$, such that, for any $p \geq \bar{p}$, we have

$$
\begin{aligned}
& \sup _{r \in[s, t]} e^{\delta p(r-s)} \mathbb{E}\left\|\Gamma_{\alpha}\left(v_{1} ; s\right)(r)-\Gamma_{\alpha}\left(v_{2} ; s\right)(r)\right\|_{\mathbb{G}}^{p} \\
& \quad \leq c_{p, 1} \frac{L_{f_{2}}^{p}}{(\alpha-\delta)^{c_{p, 2}}} \times \sup _{r \in[s, t]} e^{\delta p(r-s)} \mathbb{E}\left\|v_{1}(r)-v_{2}(r)\right\|_{\mathbb{Q}}^{p},
\end{aligned}
$$

where $L_{f_{2}}$ is the Lipschitz constant of $f_{2}$ and $c_{p, 1}$ and $c_{p, 2}$ are two suitable positive constants independent of $\alpha>0$ and $s<t$.

For the stochastic term $\Psi_{\alpha}(v ; s)(t)$, using Kunita's first inequality ([26], Theorem 4.4.23), we get 


$$
\begin{aligned}
& \mathbb{E}\left\|\Psi_{\alpha}\left(v_{1} ; s\right)(t)-\Psi_{\alpha}\left(v_{2} ; s\right)(t)\right\|_{\mathbb{H}}^{p} \leq c_{p} \mathbb{E}\left(\int_{s}^{t} \int_{\mathbb{Z}}\left\|e^{-\alpha(t-r)} e^{\gamma_{2}(t, r) A_{2}}\left[G_{2}\left(r, x, v_{1}(r), z\right)-G_{2}\left(r, x, v_{2}(r), z\right)\right]\right\|_{\mathbb{H}}^{2} v_{2_{1}}(\mathrm{~d} z) \mathrm{d} r\right)^{p / 2} \\
& \quad+c_{p} \mathbb{E} \int_{s}^{t} \int_{\mathbb{Z}}\left\|e^{-\alpha(t-r)} e^{\gamma_{2}(t, r) A_{2}}\left[G_{2}\left(r, x, v_{1}(r), z\right)-G_{2}\left(r, x, v_{2}(r), z\right)\right]\right\|_{\mathbb{H}}^{p} v_{2^{\prime}}(\mathrm{d} z) \mathrm{d} r \\
& \leq c_{p} \mathbb{E}\left(\int_{s}^{t} \int_{\mathbb{Z}} e^{-2 \alpha(t-r)}\left\|G_{2}\left(r, x, v_{1}(r), z\right)-G_{2}\left(r, x, v_{2}(r), z\right)\right\|_{\mathbb{H}}^{2} v_{2^{\prime}}(\mathrm{d} z) \mathrm{d} r\right)^{p / 2} \\
& \quad+c_{p} \mathbb{E} \int_{s}^{t} \int_{\mathbb{Z}} e^{-\alpha p(t-r)}\left\|G_{2}\left(r, x, v_{1}(r), z\right)-G_{2}\left(r, x, v_{2}(r), z\right)\right\|_{\mathbb{H}}^{p} v_{2^{\prime}}(\mathrm{d} z) \mathrm{d} r \\
& \leq c_{p} L_{g_{2}}^{p}\left(\int_{s}^{t} e^{-2(\alpha-\delta)(t-r)} e^{-2 \delta(t-s)} e^{2 \delta(r-s)} \mathbb{E}\left\|v_{1}(r)-v_{2}(r)\right\|_{\mathbb{H}}^{2} \mathrm{~d} r\right)^{p / 2} \\
& \quad+c_{p} L_{g_{2}}^{p} \int_{s}^{t} e^{-p(\alpha-\delta)(t-r)} e^{-\delta p(t-s)} e^{\delta p(r-s)} \mathbb{E}\left\|v_{1}(r)-v_{2}(r)\right\|_{\mathbb{H}}^{p} \mathrm{~d} r \\
& \leq c_{p} L_{g_{2}}^{p}\left[\left(\int_{s}^{t} e^{-2(\alpha-\delta)(t-r)} \mathrm{d} r\right)^{p / 2}+\int_{s}^{t} e^{-p(\alpha-\delta)(t-r)} \mathrm{d} r\right] \times e^{-\delta p(t-s)} \sup _{r \in[s, t]} e^{\delta p(r-s)} \mathbb{E}\left\|v_{1}(r)-v_{2}(r)\right\|_{\mathbb{H}}^{p} \\
& \leq c_{p, 1} \frac{L_{g_{2}}^{p}}{(\alpha-\delta)^{c_{p, 2}} e^{-\delta p(t-s)} \sup _{r \in[s, t]} e^{\delta p(r-s)} \mathbb{E}\left\|v_{1}(r)-v_{2}(r)\right\|_{\mathbb{H}}^{p} .}
\end{aligned}
$$

so

$$
\sup _{r \in[s, t]} e^{\delta p(r-s)} \mathbb{E}\left\|\Psi_{\alpha}\left(v_{1} ; s\right)(r)-\Psi_{\alpha}\left(v_{2} ; s\right)(r)\right\|_{\mathbb{G}}^{p} \leq c_{p, 1} \frac{L_{g_{2}}^{p}}{(\alpha-\delta)^{c_{p, 2}}} \times \sup _{r \in[s, t]} e^{\delta p(r-s)} \mathbb{E}\left\|v_{1}(r)-v_{2}(r)\right\|_{\mathbb{G}}^{p},
$$

where $L_{g_{2}}$ is the Lipschitz constant of $g_{2}$ and $c_{p, 1}$ and $c_{p, 2}$ are two suitable positive constants independent of $\alpha>0$ and $s<t$.

Moreover, using (A4), we can show that

$$
\begin{aligned}
& \sup _{r \in[s, t]} e^{\delta p(r-s)} \mathbb{E}\left\|\Gamma_{\alpha}(v ; s)(r)\right\|_{\mathbb{G}}^{p} \leq c_{p, 1} \frac{M_{f_{2}}^{p}}{(\alpha-\delta)^{c_{p, 2}}} \\
& \sup _{r \in[s, t]} e^{\delta p(r-s)}\left(1+\mathbb{E}\|v(r)\|_{\mathbb{H}}^{p}\right), \\
& \sup _{r \in[s, t]} e^{\delta p(r-s)} \mathbb{E}\left\|\Psi_{\alpha}(v ; s)(r)\right\|_{\mathbb{W}}^{p} \leq c_{p, 1} \frac{M_{g_{2}}^{p}}{(\alpha-\delta)^{c_{p, 2}}} \\
& \sup _{r \in[s, t]} e^{\delta p(r-s)}\left(1+\mathbb{E}\|v(r)\|_{\mathbb{H}}^{p}\right),
\end{aligned}
$$

where $M_{f_{2}}$ and $M_{g_{2}}$ are the linear growth constants of $f_{2}$ and $g_{2}$ and $c_{p, 1}$ and $c_{p, 2}$ are two suitable positive constants independent of $\alpha>0$ and $s<t$.

For any fixed adapted process $v$, let us introduce the problem:

$$
\begin{aligned}
\mathrm{d} \rho(t)= & \left(A_{2}(t)-\alpha\right) \rho(t) \mathrm{d} t+F_{2}(t, x, v(t)) \mathrm{d} \bar{\omega}^{Q_{2}}(t) \\
& +\int_{\mathbb{Z}} G_{2}(t, x, v(t), z) \tilde{N}_{2,}(\mathrm{~d} t, \mathrm{~d} z), \quad \rho(s)=0 .
\end{aligned}
$$

We denote that its unique mild solution is $\rho_{\alpha}(v ; s)$. This means that $\rho_{\alpha}(v ; s)$ solves the equation:

$$
\begin{aligned}
\rho_{\alpha}(v ; s)(t)= & \psi_{\alpha, 2}\left(\rho_{\alpha}(v ; s) ; s\right)(t)+\Gamma_{\alpha}(v ; s)(t) \\
& +\Psi_{\alpha}(v ; s)(t), \quad s<t<T .
\end{aligned}
$$

Due to (82) and (84), using the same argument as (5.8) in [25], it is easy to prove that for any process $v_{1}, v_{2}$, and $0<\delta<\alpha$, we have

$$
\begin{aligned}
& \sup _{r \in[s, t]} e^{\delta p(r-s)} \mathbb{E}\left\|\rho_{\alpha}\left(v_{1} ; s\right)(r)-\rho_{\alpha}\left(v_{2} ; s\right)(r)\right\|_{\mathbb{H}}^{p} \\
& \quad \leq c_{p, 1} \frac{L^{p}}{(\alpha-\delta)^{c_{p, 2}}} \times \sup _{r \in[s, t]} e^{\delta p(r-s)} \mathbb{E}\left\|v_{1}(r)-v_{2}(r)\right\|_{\mathbb{Q}}^{p},
\end{aligned}
$$

where, $L=\max \left\{L_{b_{2}}, L_{f_{2}}, L_{g_{2}}\right\}$.

Similarly, thanks to (85) and (86), for any process $v$ and $0<\delta<\alpha$, we can prove that

$$
\begin{aligned}
& \sup _{r \in[s, t]} e^{\delta p(r-s)} \mathbb{E}\left\|\rho_{\alpha}(v ; s)(r)\right\|_{\mathbb{Q}}^{p} \leq c_{p, 1} \frac{M^{p}}{(\alpha-\delta)^{c_{p, 2}}} \\
& \sup _{r \in[s, t]} e^{\delta p(r-s)} \mathbb{E}\left(1+\|v(r)\|_{\mathbb{G}}^{p}\right),
\end{aligned}
$$

where $M=\max \left\{M_{b_{2}}, M_{f_{2}}, M_{g_{2}}\right\}$. 
Lemma 4. Under (A1)-(A4), there exists $\delta>0$, such that, for any $x, y \in \mathbb{H}$ and $p \geq 1$,

$$
\mathbb{E}\left\|v^{x}(t ; s, y)\right\|_{\mathbb{Q}}^{p} \leq c_{p}\left(1+\|x\|_{\mathbb{Q}}^{p}+e^{-\delta p(t-s)}\|y\|_{\mathbb{Q}}^{p}\right), \quad s<t .
$$

Proof. We set $\Lambda_{\alpha}(t):=v^{x}(t ; s, y)-\rho_{\alpha}(t)$, where $\rho_{\alpha}(t)=\rho_{\alpha}\left(v^{x}(\cdot ; s, y) ; s\right)(t)$ is the solution of problem (87) with $v=v^{x}(\cdot ; s, y)$. Using Young's inequality, we have

$$
\begin{aligned}
& \frac{1}{p} \frac{\mathrm{d}}{\mathrm{d} t}\left\|\Lambda_{\alpha}(t)\right\|_{\mathbb{H}}^{p} \leq\left\langle\left(A_{2}(t)-\alpha\right) \Lambda_{\alpha}(t), \Lambda_{\alpha}(t)\right\rangle_{\mathbb{H}}\left\|\Lambda_{\alpha}(t)\right\|_{\mathbb{H}}^{p-2} \\
& +\left\langle B_{2}\left(t, x, \Lambda_{\alpha}(t)+\rho_{\alpha}(t)\right)-B_{2}\left(t, x, \rho_{\alpha}(t)\right), \Lambda_{\alpha}(t)\right\rangle_{\mathbb{H}}\left\|\Lambda_{\alpha}(t)\right\|_{\mathbb{M}}^{p-2} \\
& +\left\langle B_{2}\left(t, x, \rho_{\alpha}(t)\right), \Lambda_{\alpha}(t)\right\rangle_{\mathbb{H}}\left\|\Lambda_{\alpha}(t)\right\|_{\mathbb{H}}^{p-2} \leq-\alpha\left\|\Lambda_{\alpha}(t)\right\|_{\mathbb{H}}^{p} \\
& +c\left\|\Lambda_{\alpha}(t)\right\|_{\mathbb{M}}^{p}+c\left(1+\|x\|_{\mathbb{H}}+\left\|\rho_{\alpha}(t)\right\|_{\mathbb{H}}\right)\left\|\Lambda_{\alpha}(t)\right\|_{\mathbb{H}}^{p-1} \\
& \leq-\alpha\left\|\Lambda_{\alpha}(t)\right\|_{\mathbb{M}}^{p}+c_{p}\left\|\Lambda_{\alpha}(t)\right\|_{\mathbb{M}}^{p}+c_{p}\left(1+\|x\|_{\mathbb{M}}^{p}+\left\|\rho_{\alpha}(t)\right\|_{\mathbb{M}}^{p}\right) .
\end{aligned}
$$

Because $\alpha$ is large enough, we can find $\eta=\alpha-c_{p}>0$, such that

$$
\frac{\mathrm{d}}{\mathrm{d} t}\left\|\Lambda_{\alpha}(t)\right\|_{\mathbb{Q}}^{p} \leq-\eta p\left\|\Lambda_{\alpha}(t)\right\|_{\mathbb{Q}}^{p}+c_{p}\left(1+\|x\|_{\mathbb{Q}}^{p}+\left\|\rho_{\alpha}(t)\right\|_{\mathbb{E}}^{p}\right) .
$$

According to the Gronwall inequality, we have

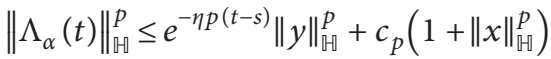

$$
\begin{aligned}
& +c_{p} \int_{s}^{t} e^{-\eta p(t-r)}\left\|\rho_{\alpha}(r)\right\|_{\mathbb{G}}^{p} \mathrm{~d} r .
\end{aligned}
$$

So, for any $p \geq 1$,

$$
\begin{aligned}
& \left\|v^{x}(t ; s, y)\right\|_{\mathbb{Q}}^{p} \leq c_{p}\left\|\rho_{\alpha}(t)\right\|_{\mathbb{Q}}^{p}+c_{p} e^{-\eta p(t-s)}\|y\|_{\mathbb{Q}}^{p}+c_{p}\left(1+\|x\|_{\mathbb{Q}}^{p}\right) \\
& +c_{p} \int_{s}^{t} e^{-\eta p(t-r)}\left\|\rho_{\alpha}(r)\right\|_{\mathbb{Q}}^{p} \mathrm{~d} r
\end{aligned}
$$

Fixing $0<\delta<\eta$, according to (90), we get

$$
\begin{aligned}
& e^{\delta p(t-s)} \mathbb{E}\left\|v^{x}(t ; s, y)\right\|_{\mathbb{G}}^{p} \leq c_{p} e^{\delta p(t-s)} \mathbb{E}\left\|\rho_{\alpha}(t)\right\|_{\mathbb{G}}^{p}+c_{p} e^{p(\delta-\eta)(t-s)}\|y\|_{\mathbb{Q}}^{p}+c_{p} e^{\delta p(t-s)}\left(1+\|x\|_{\mathbb{Q}}^{p}\right)+c_{p} \int_{s}^{t} e^{\delta p(r-s)} \mathbb{E}\left\|\rho_{\alpha}(r)\right\|_{\mathbb{G}}^{p} \mathrm{~d} r \\
& \leq c_{p, 1} \frac{M^{p}}{(\alpha-\delta)^{c_{p, 2}}} \sup _{r \in[s, t]} e^{\delta p(r-s)}\left(1+\mathbb{E}\left\|v^{x}(r ; s, y)\right\|_{\mathbb{G}}^{p}\right)+c_{p} e^{p(\delta-\eta)(t-s)}\|y\|_{\mathbb{Q}}^{p}+c_{p} e^{\delta p(t-s)}\left(1+\|x\|_{\mathbb{Q}}^{p}\right) \\
& \quad+c_{p, 1} \frac{M^{p}}{(\alpha-\delta)^{c_{p, 2}}} \int_{s}^{t} \sup _{r \in[s, t]} e^{\delta p(r-s)}\left(1+\mathbb{E}\left\|v^{x}(r ; s, y)\right\|_{\mathbb{G}}^{p}\right) \mathrm{d} r .
\end{aligned}
$$

Taking $\alpha_{1}=\left(2 c_{p, 1} M^{p}\right)^{1 / c_{p, 2}}+\delta$, when $\alpha \geq \alpha_{1}$, we have

$$
\begin{aligned}
& \sup _{r \in[s, t]} e^{\delta p(r-s)} \mathbb{E}\left\|v^{x}(r ; s, y)\right\|_{\mathbb{Q}}^{p} \leq c_{p}\|y\|_{\mathbb{Q}}^{p}+c_{p} e^{\delta p(t-s)}\left(1+\|x\|_{\mathbb{G}}^{p}\right) \\
& \quad+\int_{s}^{t} \sup _{r \in[s, t]} e^{\delta p(r-s)} \mathbb{E}\left\|v^{x}(r ; s, y)\right\|_{\mathbb{G}}^{p} \mathrm{~d} r .
\end{aligned}
$$

Due to the Gronwall lemma, we have

$$
\sup _{r \in[s, t]} e^{\delta p(r-s)} \mathbb{E}\left\|v^{x}(r ; s, y)\right\|_{\mathbb{G}}^{p} \leq c_{p}\|y\|_{\mathbb{Q}}^{p}+c_{p} e^{\delta p(t-s)}\left(1+\|x\|_{\mathbb{Q}}^{p}\right) .
$$

Hence, we get (91).

Lemma 5. Under (A1)-(A4), for any $t \in \mathbb{R}$ and $x, y \in \mathbb{H}$, for all $p \geq 1$, there exists $\eta^{x}(t) \in L^{p}(\Omega ; \mathbb{H})$ such that

$$
\lim _{s \longrightarrow-\infty} \mathbb{E}\left\|v^{x}(t ; s, y)-\eta^{x}(t)\right\|_{\mathbb{H}}^{p}=0
$$
that

Moreover, for any $p \geq 1$, there exists some $\delta_{p}>0$, such $\mathbb{E}\left\|v^{x}(t ; s, y)-\eta^{x}(t)\right\|_{\mathbb{Q}}^{p} \leq c_{p} e^{-\delta_{p}(t-s)}\left(1+\|x\|_{\mathbb{Q}}^{p}+\|y\|_{\mathbb{Q}}^{p}\right)$.

Finally, $\eta^{x}$ is a mild solution in $\mathbb{R}$ of (79).

Proof. Fix $h>0$ and define

$$
\rho(t)=v^{x}(t ; s, y)-v^{x}(t ; s-h, y), \quad s<t .
$$

We know that $\rho(t)$ is the unique mild solution of the problem: 


$$
\begin{aligned}
& \left\{\begin{array}{l}
\mathrm{d} \rho(t)=\left[\left(A_{2}(t)-\alpha\right) \rho(t)+B_{2}\left(t, x, v^{x}(t ; s, y)\right)-B_{2}\left(t, x, v^{x}(t ; s-h, y)\right)\right] \mathrm{d} t \\
+\left[F_{2}\left(t, x, v^{x}(t ; s, y)\right)-F_{2}\left(t, x, v^{x}(t ; s-h, y)\right)\right] \mathrm{d} \bar{w}^{Q_{2}}(t) \\
+\int_{\mathbb{Z}}\left[G_{2}\left(t, x, v^{x}(t ; s, y)\right)-G_{2}\left(t, x, v^{x}(t ; s-h, y)\right)\right] \tilde{N}_{2,}(\mathrm{~d} t, \mathrm{~d} z), \\
\rho(s)=y-v^{x}(s ; s-h, y),
\end{array}\right. \\
& \rho(t)=U_{\alpha, 2}(t, s)\left(y-v^{x}(s ; s-h, y)\right)+\psi_{\alpha, 2}(\rho ; s)(t) \\
& +\int_{s}^{t} U_{\alpha, 2}(t, r)\left[B_{2}\left(r, x, v^{x}(r ; s, y)\right)-B_{2}\left(r, x, v^{x}(r ; s-h, y)\right)\right] \mathrm{d} r \\
& +\int_{s}^{t} U_{\alpha, 2}(t, r)\left[F_{2}\left(r, x, v^{x}(r ; s, y)\right)-F_{2}\left(r, x, v^{x}(r ; s-h, y)\right)\right] \mathrm{d} \bar{w}^{Q_{2}}(r) \\
& +\int_{s}^{t} \int_{\mathbb{Z}} U_{\alpha, 2}(t, r)\left[G_{2}\left(r, x, v^{x}(r ; s, y)\right)-G_{2}\left(r, x, v^{x}(r ; s-h, y)\right)\right] \tilde{N}_{2,}(\mathrm{~d} r, \mathrm{~d} z) .
\end{aligned}
$$

Multiply both sides of the above equation by $e^{\delta p(t-s)}$. Because $\alpha$ large enough, according to Lemma 2.4 in [25], we have

$$
\begin{aligned}
e^{\delta p(t-s)} \mathbb{E}\|\rho(t)\|_{\mathbb{H}}^{p} \leq & c_{p} e^{(\delta-\alpha) p(t-s)} \mathbb{E}\left\|e^{\gamma_{2}(t, s) A_{2}}\left(y-v^{x}(s ; s-h, y)\right)\right\|_{\mathbb{E}}^{p} \\
& +c_{p} \mathbb{E}\left\|\int_{s}^{t} e^{\gamma_{2}(t, r) A_{2}} e^{(\delta-\alpha)(t-r)} e^{\delta(r-s)}\left[B_{2}\left(r, x, v^{x}(r ; s, y)\right)-B_{2}\left(r, x, v^{x}(r ; s-h, y)\right)\right] \mathrm{dr}\right\|_{\mathbb{Q}}^{p} \\
& +c_{p} \mathbb{E}\left\|\int_{s}^{t} e^{\gamma_{2}(t, r) A_{2}} e^{(\delta-\alpha)(t-r)} e^{\delta(r-s)}\left[F_{2}\left(r, x, v^{x}(r ; s, y)\right)-F_{2}\left(r, x, v^{x}(r ; s-h, y)\right)\right] \mathrm{d} \bar{w}^{Q_{2}}(r)\right\|_{\mathbb{Q}}^{p} \\
& +c_{p} \mathbb{E}\left\|\int_{s}^{t} \int_{\mathbb{Z}} e^{\gamma_{2}(t, r) A_{2}} e^{(\delta-\alpha)(t-r)} e^{\delta(r-s)}\left[G_{2}\left(r, x, v^{x}(r ; s, y)\right)-G_{2}\left(r, x, v^{x}(r ; s-h, y)\right)\right] \tilde{N}_{2}(\mathrm{~d} r, \mathrm{~d} z)\right\|_{\mathbb{U}}^{p}:=\sum_{i=1}^{4} \mathscr{J}_{t}^{i} .
\end{aligned}
$$

According to Lemma 3.1 in [12], we know that, for any $J \in \mathscr{L}\left(L^{\infty}(D), \mathbb{H}\right) \cap \mathscr{L}\left(\mathbb{H}, L^{1}(D)\right)$ with $J=J^{*}$ and $s \geq 0$, we have

$$
\left\|e^{s A_{i}} J Q_{i}\right\|_{2}^{2} \leq K_{i} s^{-\beta_{i}\left(\rho_{i}-2\right) / \rho_{i}} e^{-\left(\alpha\left(\rho_{i}+2\right) / \rho_{i}\right) s}\|J\|_{\mathscr{L}\left(L^{\infty}(D), \mathbb{W}\right)}^{2},
$$

(104)

$$
K_{i}=\left(\frac{\beta_{i}}{e}\right)^{\beta_{i}\left(\rho_{i}-2\right) / \rho_{i}} \zeta_{i}^{\left(\rho_{i}-2\right) / \rho_{i}} \kappa_{i}^{2 / \rho_{i}}
$$

Taking $\bar{p}>1$, such that $\bar{p} \beta_{2}\left(\rho_{2}-2\right) /\left[\rho_{2}(\bar{p}-2)\right]<1$. Then, using the Burkholder-Davis-Gundy inequality and Kunita's first inequality, we can get that, for any $p \geq \bar{p}$ and $0<\delta<\alpha$, it yields

where

$$
\mathscr{I}_{t}^{2} \leq c_{p} L_{b_{2}}^{p} \sup _{r \in[s, t]} e^{\delta p(r-s)} \mathbb{E}\|\rho(r)\|_{\mathbb{Q}}^{p} \cdot\left(\int_{s}^{t} e^{(\delta-\alpha)(t-r)} \mathrm{d} r\right)^{p} \leq c_{p} \frac{L_{b_{2}}^{p}}{(\alpha-\delta)^{p}} \sup _{r \in[s, t]} e^{\delta p(r-s)} \mathbb{E}\|\rho(r)\|_{\mathbb{G}}^{p},
$$




$$
\begin{aligned}
& \mathscr{J}_{t}^{3} \leq c_{p} \mathbb{E}\left(\int_{s}^{t}\left\|e^{\gamma_{2}(t, r) A_{2}} e^{(\delta-\alpha)(t-r)} e^{\delta(r-s)}\left[F_{2}\left(r, x, v^{x}(r ; s, y)\right) \quad-F_{2}\left(r, x, v^{x}(r ; s-h, y)\right)\right] Q_{2}\right\|_{2}^{2} \mathrm{~d} r\right)^{p / 2} \\
& \leq c_{p} L_{f_{2}}^{p} K_{2}^{p / 2}\left(\int_{s}^{t} e^{2(\delta-\alpha)(t-r)} \gamma_{2}(t, r)^{-\left(\beta_{2}\left(\rho_{2}-2\right) / \rho_{2}\right)} e^{-\alpha\left(\rho_{2}+2\right) / \rho_{2} \gamma_{2}(t, r)} \mathrm{d} r\right)^{p / 2} \\
& \times \sup _{r \in[s, t]} e^{\delta p(r-s)} \mathbb{E}\|\rho(r)\|_{\mathbb{Q}}^{p} \leq c_{p} L_{f_{2}}^{p} \sup _{r \in[s, t]} e^{\delta p(r-s)} \mathbb{E}\|\rho(r)\|_{\mathbb{Q}}^{p} \cdot\left(\int_{s}^{t} e^{2(\delta-\alpha)(t-r)}\left[\gamma_{0}(t-r)\right]^{-\left(\beta_{2}\left(\rho_{2}-2\right) / \rho_{2}\right)} \mathrm{d} r\right)^{p / 2} \\
& \leq c_{p} L_{f_{2}}^{p} \sup _{r \in[s, t]} e^{\delta p(r-s)} \mathbb{E}\|\rho(r)\|_{\mathbb{G}}^{p} \cdot\left(\int_{0}^{t-s} r^{-\left(\beta_{2}\left(\rho_{2}-2\right) / \rho_{2}\right)(p /(p-2))} \mathrm{d} r\right)^{(p-2) / 2}\left(\int_{0}^{t-s} e^{-p(\alpha-\delta) r} \mathrm{~d} r\right) \\
& \leq c_{p} \frac{L_{f_{2}}^{p}}{\alpha-\delta} \sup _{r \in[s, t]} e^{\delta p(r-s)} \mathbb{E}\|\rho(r)\|_{\mathbb{R}}^{p}, \\
& \mathscr{G}_{t}^{4} \leq c_{p} \mathbb{E}\left(\int_{s}^{t} \int_{\mathbb{Z}}\left\|e^{\gamma_{2}(t, r) A_{2}} e^{(\delta-\alpha)(t-r)} e^{\delta(r-s)}\left[G_{2}\left(r, x, v^{x}(r ; s, y)\right)-G_{2}\left(r, x, v^{x}(r ; s-h, y)\right)\right]\right\|_{\mathbb{Q}}^{2} v_{2,}(\mathrm{~d} z) \mathrm{d} r\right)^{p / 2} . \\
& +c_{p} \mathbb{E} \int_{s}^{t} \int_{\mathbb{Z}}\left\|e^{\gamma_{2}(t, r) A_{2}} e^{(\delta-\alpha)(t-r)} e^{\delta(r-s)}\left[G_{2}\left(r, x, v^{x}(r ; s, y)\right)-G_{2}\left(r, x, v^{x}(r ; s-h, y)\right)\right]\right\|_{\mathbb{E}}^{p} v_{2,}(\mathrm{~d} z) \mathrm{d} r \\
& \leq c_{p} L_{g_{2}}^{p} \sup _{r \in[s, t]} e^{\delta p(r-s)} \mathbb{E}\|\rho(r)\|_{\mathbb{\square}}^{p} \cdot\left[\left(\int_{0}^{t-s} e^{-2(\alpha-\delta) r} \mathrm{~d} r\right)^{p / 2}+\int_{0}^{t-s} e^{-p(\alpha-\delta) r} \mathrm{~d} r\right] \leq c_{p} \frac{L_{g_{2}}^{p}}{(\alpha-\delta)^{c_{p}}} \sup _{r \in[s, t]} e^{\delta p(r-s)} \mathbb{E}\|\rho(r)\|_{\mathbb{\square}}^{p} .
\end{aligned}
$$

Hence, we have

$$
\begin{aligned}
& \sup _{r \in[s, t]} e^{\delta p(r-s)} \mathbb{E}\|\rho(r)\|_{\mathbb{a}}^{p} \leq c_{p}\left\|y-v^{x}(s ; s-h, y)\right\|_{\mathbb{W}}^{p} \\
& \quad+c_{p, 1} \frac{L^{p}}{(\alpha-\delta)^{c_{p, 2}}} \sup _{r \in[s, t]} e^{\delta p(r-s)} \mathbb{E}\|\rho(r)\|_{\mathbb{Q}}^{p} .
\end{aligned}
$$

Therefore, for $\alpha>0$ large enough, we can find $0<\bar{\delta}_{p}<\alpha$, such that

$$
c_{p, 1} \frac{L}{\left(\alpha-\bar{\delta}_{p}\right)^{c_{p, 2}}}<1 .
$$

This implies that

$$
\sup _{r \in[s, t]} e^{p \bar{\delta}_{p}(r-s)} \mathbb{E}\|\rho(r)\|_{\mathbb{Q}}^{p} \leq c_{p}\left\|y-v^{x}(s ; s-h, y)\right\|_{\mathbb{Q}}^{p} .
$$

Let $\delta_{p}=p \bar{\delta}_{p}$, thanks to Lemma 4 , we have

$$
\begin{aligned}
& \mathbb{E}\left\|v^{x}(t ; s, y)-v^{x}(t ; s-h, y)\right\|_{\mathbb{Q}}^{p} \\
& \leq c_{p} e^{-\delta_{p}(t-s)}\left\|y-v^{x}(s ; s-h, y)\right\|_{\mathbb{Q}}^{p} \\
& \leq c_{p} e^{-\delta_{p}(t-s)}\left(1+\|x\|_{\mathbb{Q}}^{p}+\|y\|_{\mathbb{Q}}^{p}+e^{-\delta \mathrm{ph}}\|y\|_{\mathbb{Q}}^{p}\right) .
\end{aligned}
$$

Because $L^{p}(\Omega ; \mathbb{H})$ has completeness, for any $p \geq \bar{p}$, let $s \longrightarrow-\infty$ in $(111)$, there exists $\eta^{x}(t) \in L^{p}(\Omega ; \mathbb{U})$ such that (99) holds. Then, if we let $h \longrightarrow \infty$ in (111), we obtain (100). Using the Hölder inequality, we can get (99), and (100) holds for any $p<\bar{p}$.

If we take $y_{1}, y_{2} \in \mathbb{U}$, use the same arguments for $v^{x}\left(t ; s, y_{1}\right)-v^{x}\left(t ; s, y_{2}\right), s<t$, we have

$$
\mathbb{E}\left\|v^{x}\left(t ; s, y_{1}\right)-v^{x}\left(t ; s, y_{2}\right)\right\|_{\mathbb{H}}^{p} \leq c_{p} e^{-\delta_{p}(t-s)}\left\|y_{1}-y_{2}\right\|_{\mathbb{H}}^{p}, \quad s<t .
$$

Let $s \longrightarrow-\infty$, this means that the limit $\eta^{x}(t)$ does not depend on the initial condition $y \in \mathbb{H}$.

Finally, we prove that $\eta^{x}(t)$ is a mild solution of (79). Due to the limit $\eta^{x}(t)$ does not depend on the initial condition, we can let initial condition $y=0$. For any $s<t$ and $h>0$, we have

$$
\begin{aligned}
v^{x}(t ; s-h, 0)= & U_{\alpha, 2}(t, s) v^{x}(s ; s-h, 0)+\psi_{\alpha, 2}\left(v^{x}(\cdot ; s-h, 0) ; s\right)(t) \\
& +\int_{s}^{t} U_{\alpha, 2}(t, r) B_{2}\left(r, x, v^{x}(r ; s-h, 0)\right) \mathrm{d} r \\
& +\int_{s}^{t} U_{\alpha, 2}(t, r) F_{2}\left(r, x, v^{x}(r ; s-h, 0)\right) \mathrm{d} \bar{w}^{Q_{2}}(r) \\
& +\int_{s}^{t} \int_{\mathbb{Z}} U_{\alpha, 2}(t, r) G_{2}\left(r, x, v^{x}(r ; s-h, 0), z\right) \tilde{N}_{2,}(\mathrm{~d} r, \mathrm{~d} z) .
\end{aligned}
$$

Let $h \longrightarrow \infty$ on both sides, due to (99), we can get, for any $s<t$, have

$$
\begin{aligned}
\eta^{x}(t)= & U_{\alpha, 2}(t, s) \eta^{x}(s)+\psi_{\alpha, 2}\left(\eta^{x} ; s\right)(t) \\
& +\int_{s}^{t} U_{\alpha, 2}(t, r) B_{2}\left(r, x, \eta^{x}(r)\right) \mathrm{d} r \\
& +\int_{s}^{t} U_{\alpha, 2}(t, r) F_{2}\left(r, x, \eta^{x}(r)\right) \mathrm{d} \bar{w}^{Q_{2}}(r) \\
& +\int_{s}^{t} \int_{\mathbb{Z}} U_{\alpha, 2}(t, r) G_{2}\left(r, x, \eta^{x}(r), z\right) \tilde{N}_{2,}(\mathrm{~d} r, \mathrm{~d} z) .
\end{aligned}
$$

This means that $\eta^{x}(t)$ is a mild solution of (79).

For any $t \in \mathbb{R}$ and $x \in \mathbb{H}$, we denote that the law of the random variable $\eta^{x}(t)$ is $\mu_{t}^{x}$, and we introduce the transition evolution operator:

$$
P_{s, t}^{x} \varphi(y)=\mathbb{E} \varphi\left(v^{x}(t ; s, y)\right), \quad s<t, y \in \mathbb{H},
$$


where $\varphi \in \mathscr{B}_{b}(\mathbb{\square})$.

Due to (91) and (99), for any $p \geq 1$, we have

$$
\sup _{t \in \mathbb{R}} \mathbb{E}\left\|\eta^{x}(t)\right\|_{\mathbb{W}}^{p} \leq c_{p}\left(1+\|x\|_{\mathbb{H}}^{p}\right), \quad x \in \mathbb{H},
$$

so that

$$
\sup _{t \in \mathbb{R}} \int_{\mathbb{H}}\|y\|_{\mathbb{H}}^{p} \mu_{t}^{x}(d y) \leq c_{p}\left(1+\|x\|_{\mathbb{H}}^{p}\right), \quad x \in \mathbb{M} .
$$

According to the above conclusion, by using the same arguments as ([25], Proposition 5.3), we know that the family $\left\{\mu_{t}^{x}\right\}_{t \in \mathbb{R}}$ defines an evolution system of probability measures on $\mathbb{U}$ for equation (76). This means that, for any $t \in \mathbb{R}, \mu_{t}^{x}$ is a probability measure on $\mathbb{Z}$, and it holds that

$$
\int_{\mathbb{H}} P_{s, t}^{x} \varphi(y) \mu_{s}^{x}(\mathrm{~d} y)=\int_{\mathbb{H}} \varphi(y) \mu_{t}^{x}(\mathrm{~d} y), \quad s<t,
$$

for every $\varphi \in \mathscr{C}_{b}(\mathbb{H})$. Moreover, we also have

$$
\left|P_{s, t}^{x} \varphi(y)-\int_{\mathbb{H}} \varphi(y) \mu_{t}^{x}(\mathrm{~d} y)\right| \leq c e^{-\delta_{1}(t-s)}\left(1+\|x\|_{\mathbb{H}}\right) .
$$

Lemma 6. Under (A1)-(A4), the family of measures

$$
\Lambda:=\left\{\mu_{t}^{x}: t \in \mathbb{R}, x \in \mathbb{W}\right\}
$$

is tight in $\mathscr{P}(D([0, T] ; \mathbb{U}))$.

Proof. According to the definition of $v^{x}(\cdot ; s, 0)$, for any $t>s$ and $h>0$, we have

$$
\begin{aligned}
& v^{x}(t+h ; s, 0)-v^{x}(t ; s, 0)=\left(U_{\alpha, 2}(t+h, t)-I\right) v^{x}(t ; s, 0) \\
& +\psi_{\alpha, 2}\left(v^{x}(\cdot ; s, 0) ; t\right)(t+h)+\int_{t}^{t+h} U_{\alpha, 2}(t+h, r) \\
& \cdot B_{2}\left(r, x, v^{x}(r ; s, 0)\right) \mathrm{d} r+\int_{t}^{t+h} U_{\alpha, 2}(t+h, r) \\
& \cdot F_{2}\left(r, x, v^{x}(r ; s, 0)\right) \mathrm{d} \bar{w}^{Q_{2}}(r)+\int_{t}^{t+h} \\
& \cdot \int_{\mathbb{Z}} U_{\alpha, 2}(t+h, r) G_{2}\left(r, x, v^{x}(r ; s, 0), z\right) \widetilde{N}_{2,}(\mathrm{~d} r, \mathrm{~d} z) .
\end{aligned}
$$

Due to the assumption (A4), we know that the mappings $B_{2}, F_{2}$, and $G_{2}$ are linearly growing. For any $t>s, 0<h<1$, and $x \in \mathbb{U}$, analogous to the proof of Lemma 3 and using the estimate (91), we can get

$$
\mathbb{E}\left\|v^{x}(t+h ; s, 0)-v^{x}(t ; s, 0)\right\|_{\mathscr{W}}^{p} \leq c_{p}\left(h^{\kappa(p)}+h\right)\left(1+\|x\|_{\bullet \rightarrow}^{p}\right),
$$

where $\kappa(p)$ is a function of $p$ and satisfies $\kappa(p)>0$.

From Lemma 5, we know that the limit $\eta^{x}(t)$ does not depend on the initial condition, and we can get

$$
\begin{aligned}
& \mathbb{E}\left\|\eta^{x}(t+h)-\eta^{x}(t)\right\|_{\mathbb{H}}^{p} \leq \mathbb{E}\left\|\eta^{x}(t+h)-v^{x}(t+h ; s, 0)\right\|_{\mathbb{H}}^{p} \\
& \quad+\mathbb{E}\left\|v^{x}(t+h ; s, 0)-v^{x}(t ; s, 0)\right\|_{\mathbb{H}}^{p}+\mathbb{E}\left\|v^{x}(t ; s, 0)-\eta^{x}(t)\right\|_{\mathbb{H}}^{p} .
\end{aligned}
$$

Then, thanks to (100) and (122), let $s \longrightarrow \infty$, we have

$$
\mathbb{E}\left\|\eta^{x}(t+h)-\eta^{x}(t)\right\|_{\mathbb{G}}^{p} \leq c_{p}\left(h^{\kappa(p)}+h\right)\left(1+\|x\|_{\mathbb{G}}^{p}\right) .
$$

Moreover, we have proved that the family $\left\{\mu_{t}^{x}\right\}_{t \in \mathbb{R}}$ defines an evolution system of probability measures for (76). By using Chebyshev's inequality and (116), it yields

$$
\begin{aligned}
& \lim _{n \longrightarrow \infty} \mu_{t}^{x}\left(\eta_{x}(t):\left\|\eta_{x}(t)\right\|_{\mathbb{\uplus}} \geq n\right) \leq \lim _{n \longrightarrow \infty} \frac{\mathbb{E}\left\|\eta^{x}(t)\right\|_{\mathbb{H}}^{2}}{n^{2}} \\
& \quad \leq \lim _{n \longrightarrow \infty} c_{2} \frac{1+\|x\|_{\mathbb{W}}^{2}}{n^{2}}=0 .
\end{aligned}
$$

In addition, for any $t>s, h>0$, and fixed $\epsilon>0$, according to the (124), we have

$$
\begin{aligned}
& \lim _{h \longrightarrow 0} \mu_{t}^{x}\left(\eta_{x}(t):\left\|\eta_{x}(t+h)-\eta_{x}(t)\right\|_{\mathbb{H}} \geq \epsilon\right) \\
& \leq \lim _{h \longrightarrow 0} \frac{\mathbb{E}\left\|\eta^{x}(t+h)-\eta^{x}(t)\right\|_{\mathbb{H}}^{2}}{\epsilon^{2}} \\
& \leq \lim _{h \longrightarrow 0} c_{2} \frac{\left(h^{\kappa(2)}+h\right)\left(1+\|x\|_{\mathbb{H}}^{2}\right)}{\epsilon^{2}}=0 .
\end{aligned}
$$

According to Theorem 13.2 in [43], (125) and (126) imply that the family of measures

$$
\Lambda:=\left\{\mu_{t}^{x}: t \in \mathbb{R}, x \in \mathbb{E}\right\},
$$

is tight in $\mathscr{P}(D([0, T] ; \mathbb{U}))$.

In order to get the averaged equation, we must ensure the existence of the averaged coefficient $\bar{B}_{1}$. So, we need the evolution family of measures satisfying some nice properties. We give the following assumption:

(a) The functions $\gamma_{2}: \mathbb{R} \longrightarrow(0, \infty)$ and $l_{2}: \mathbb{R} \times \mathcal{O} \longrightarrow \mathbb{R}^{d}$ are periodic, with the same period.

(b) The families of functions

$$
\begin{aligned}
\mathbf{B}_{1, R} & :=\left\{b_{1}(\cdot, \xi, \sigma): \quad \xi \in \mathcal{O}, \sigma \in B_{\mathbb{R}^{2}}(R)\right\}, \\
\mathbf{B}_{2, R} & :=\left\{b_{2}(\cdot, \xi, \sigma): \quad \xi \in \mathcal{O}, \sigma \in B_{\mathbb{R}^{2}}(R)\right\}, \\
\mathbf{F}_{R} & :=\left\{f_{2}(\cdot, \xi, \sigma): \quad \xi \in \mathcal{O}, \sigma \in B_{\mathbb{R}^{2}}(R)\right\}, \\
\mathbf{G}_{R} & :=\left\{g_{2}(\cdot, \xi, \sigma, z): \quad \xi \in \mathcal{O}, \sigma \in B_{\mathbb{R}^{2}}(R), z \in \mathbb{Z}\right\}
\end{aligned}
$$

are uniformly almost periodic for any $R>0$.

Remark 4. Similar with the proof of Lemma 6.2 in [25], we get that, under (A5), for any $R>0$, the families of functions

$$
\begin{aligned}
& \left\{B_{1}(\cdot, x, y):(x, y) \in B_{\mathbb{H} \times \mathbb{H}}(R)\right\}, \\
& \left\{B_{2}(\cdot, x, y):(x, y) \in B_{\mathbb{W} \times \mathbb{H}}(R)\right\}, \\
& \left\{F_{2}(\cdot, x, y):(x, y) \in B_{\mathbb{H} \times \mathbb{\sharp}}(R)\right\}, \\
& \left\{G_{2}(\cdot, x, y, z):(x, y, z) \in B_{\mathbb{H} \times \mathbb{H}}(R) \times \mathbb{Z}\right\},
\end{aligned}
$$


are uniformly almost periodic.

As we know above, $A_{2}(\cdot)$ is periodic, Remark 4 holds, and the family of measures $\Lambda$ is tight. By proceeding as [45], we can prove that the mapping

$$
t \longmapsto \mu_{t}^{x}, \quad t \in \mathbb{R}, x \in \mathbb{H},
$$

is almost periodic.

\section{The Averaged Equation}

Lemma 7. Under (A1)-(A5), for any compact set $\mathbb{K} \subset \mathbb{M}$, the family of functions

$$
\left\{t \in \mathbb{R} \longmapsto \int_{\mathbb{Q}} B_{1}(t, x, y) \mu_{t}^{x}(\mathrm{~d} y), \quad x \in \mathbb{K}\right\},
$$

is uniformly almost periodic.

Proof. As $\mathbb{K}$ is a compact set in $\mathbb{H}$, so it is bounded and there exist some $R>0$ such that $\mathbb{K} \subset B_{\mathbb{\square}}(R)$. That is, for any $x \in \mathbb{K}$, we have $\|x\| \leq R$.

Now, let us define

$$
\Phi(t, x)=\int_{\mathbb{U}} B_{1}(t, x, y) \mu_{t}^{x}(\mathrm{~d} y), \quad(t, x) \in \mathbb{R} \times \mathbb{M} .
$$

Then, for any $t, \tau \in \mathbb{R}, n \in \mathbb{N}$ and $x \in \mathbb{K}$, according to assumption (A3) and equation (117), we can get

$$
\begin{aligned}
|\Phi(t+\tau, x)-\Phi(t, x)| \leq & \left|\int_{\|y\|_{\mathbb{H}} \leq n} B_{1}(t+\tau, x, y) \mu_{t+\tau}^{x}(\mathrm{~d} y)-\int_{\|y\|_{\mathbb{H}} \leq n} B_{1}(t+\tau, x, y) \mu_{t}^{x}(\mathrm{~d} y)\right| \\
& +\left|\int_{\|y\|_{\mathbb{H}}>n} B_{1}(t+\tau, x, y) \mu_{t+\tau}^{x}(\mathrm{~d} y)\right|+\left|\int_{\|y\|_{\mathbb{H}}>n} B_{1}(t+\tau, x, y) \mu_{t}^{x}(\mathrm{~d} y)\right| \\
& +\left|\int_{\mathbb{U}} B_{1}(t+\tau, x, y) \mu_{t}^{x}(\mathrm{~d} y)-\int_{\mathbb{U}} B_{1}(t, x, y) \mu_{t}^{x}(\mathrm{~d} y)\right| \\
\leq & \sup _{x \in \mathbb{K},\|y\|_{\mathbb{H}} \leq n}\left\|B_{1}(t+\tau, x, y)\right\|_{\mathbb{U}}\left|\int_{\mathbb{U}}\left(\mu_{t+\tau}^{x}-\mu_{t}^{x}\right)(\mathrm{d} y)\right| \\
& +\frac{c(1+\|x\|)}{n}+\left|\int_{\mathbb{H}}\left\|B_{1}(t+\tau, x, y)-B_{1}(t, x, y)\right\|_{\mathbb{H} \mathbb{R}} \mu_{t}^{x}(\mathrm{~d} y)\right| .
\end{aligned}
$$

Fixed some $\bar{n}$ such that $c(1+\|x\|) / \bar{n} \leq \epsilon / 3$. Then, for any $t \in \mathbb{R}$, due to the mapping $t \longmapsto \mu_{t}^{x}$ and the families of functions $B_{1}(\cdot, x, y)$ are almost periodic, we can find some $\tau \in \mathbb{R}$ such that

$$
|\Phi(t+\tau, x)-\Phi(t, x)|<\epsilon .
$$

So, the function $\Phi(\cdot, x)$ is almost periodic for any $x \in \mathbb{K}$.

By preceding as in Lemma 5 , we can get that, under (A1)-(A4), for any fixed $x_{1}, x_{2} \in \mathbb{K}$ and $p \geq 1$, there exists $c_{p}>0$ such that

$$
\sup _{s<t} \mathbb{E}\left\|v^{x_{1}}(t ; s, 0)-v^{x_{2}}(t ; s, 0)\right\|_{\mathbb{a}}^{p} \leq c_{p}\left\|x_{1}-x_{2}\right\|_{\mathbb{Q}}^{p} .
$$

According to (99) and (100), let $s \longrightarrow 0$, it yields

$$
\sup _{t \in \mathbb{R}} \mathbb{E}\left\|\eta^{x_{1}}(t)-\eta^{x_{2}}(t)\right\|_{\mathbb{R}}^{p} \leq c_{p}\left\|x_{1}-x_{2}\right\|_{\mathbb{G}}^{p} .
$$

Hence, thanks to (A3) and (136), we can conclude that, for any $x_{1}, x_{2} \in \mathbb{K}$, we have

$$
\begin{aligned}
\left\|\Phi\left(t, x_{1}\right)-\Phi\left(t, x_{2}\right)\right\|_{\mathbb{H}} & \leq \mathbb{E}\left\|B_{1}\left(t, x_{1}, \eta^{x_{1}}(t)\right)-B_{1}\left(t, x_{2}, \eta^{x_{2}}(t)\right)\right\|_{\mathbb{H}} \\
& \leq c\left(\left\|x_{1}-x_{2}\right\|_{\mathbb{H}}+\left(\mathbb{E}\left\|\eta^{x_{1}}(t)-\eta^{x_{2}}(t)\right\|_{\mathbb{H}}^{2}\right)^{1 / 2}\right) \\
& \leq c\left\|x_{1}-x_{2}\right\|_{\mathbb{H}} .
\end{aligned}
$$

This means that the family of functions $\{\Phi(t, \cdot): t \in \mathbb{R}\}$ is equicontinuous about $x$.

In view of Theorem 2.10 in [32], the above conclusions indicate that $\{\Phi(\cdot, x): x \in \mathbb{K}\}$ is uniformly almost periodic. Hence, the proof is complete.

According to Theorem 3.4 in [25], we define

$$
\bar{B}_{1}(x):=\lim _{T \longrightarrow \infty} \frac{1}{T} \int_{0}^{T} \int_{\mathbb{U}} B_{1}(t, x, y) \mu_{t}^{x}(\mathrm{~d} y) \mathrm{d} t, \quad x \in \mathbb{H} .
$$

And, thanks to (A3) and (117), we have that

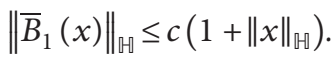

Lemma 8. Under (A1)-(A5), for any $T>0, s, \sigma \in \mathbb{R}$, and $x, y \in \mathbb{H}$,

$$
\begin{gathered}
\mathbb{E}\left\|\frac{1}{T} \int_{s}^{s+T} B_{1}\left(t, x, v^{x}(t ; s, y)\right) \mathrm{d} t-\bar{B}_{1}(x)\right\|_{\mathbb{G}} \\
\leq \frac{c}{T}\left(1+\|x\|_{\mathbb{Q}}+\|y\|_{\mathbb{Q}}\right)+\alpha(T, x),
\end{gathered}
$$




$$
\sup _{T>0} \alpha(T, x) \leq c\left(1+\|x\|_{\mathbb{H}}\right), \quad x \in \mathbb{H} .
$$

And, for any compact set $\mathbb{K} \subset \mathbb{H}$, we have

$$
\lim _{T \rightarrow \infty} \sup _{x \in \mathbb{K}} \alpha(T, x)=0
$$

Proof. We denote

$$
\psi^{x} B_{1}(t, y):=B_{1}(t, x, y)-\int_{\mathbb{U}} B_{1}(t, x, w) \mu_{t}^{x}(\mathrm{~d} w) .
$$

So,

$$
\begin{aligned}
\mathbb{E}\left(\frac{1}{T} \int_{s}^{s+T}\left[B_{1}\left(t, x, v^{x}(t ; s, y)\right)-\int_{\mathbb{H}} B_{1}(t, x, w) \mu_{t}^{x}(\mathrm{~d} w)\right] \mathrm{d} t\right)^{2} & =\frac{2}{T^{2}} \int_{s}^{s+T} \int_{r}^{s+T} \mathbb{E}\left[\psi^{x} B_{1}\left(r, v^{x}(r ; s, y)\right) \psi^{x} B_{1}\left(t, v^{x}(t ; s, y)\right)\right] \mathrm{d} t \mathrm{~d} r \\
& =\frac{2}{T^{2}} \int_{s}^{s+T} \int_{r}^{s+T} \mathbb{E}\left[\psi^{x} B_{1}\left(r, v^{x}(r ; s, y)\right) P_{r, t}^{x} \psi^{x} B_{1}\left(r, v^{x}(r ; s, y)\right)\right] \mathrm{d} t \mathrm{~d} r \\
& \leq \frac{2}{T^{2}} \int_{s}^{s+T} \int_{r}^{s+T}\left(\mathbb{E} \mid \psi^{x} B_{1}\left(r, v^{x}(r ; s, y)\right)^{2}\right)^{1 / 2}\left(\mathbb{E}\left|P_{r, t}^{x} \psi^{x} B_{1}\left(r, v^{x}(r ; s, y)\right)\right|^{2}\right)^{1 / 2} \mathrm{~d} t \mathrm{~d} r .
\end{aligned}
$$

Due to (A3), (91), and (117), we have

$$
\begin{aligned}
\mathbb{E}\left|\psi^{x} B_{1}\left(r, v^{x}(r ; s, y)\right)\right|^{2} & \leq c \mathbb{E}\left\|B_{1}\left(r, x, v^{x}(r ; s, y)\right)\right\|_{\mathbb{Q}}^{2}+c \mathbb{E}\left(\int_{\mathbb{M}}\left\|B_{1}(r, x, w)\right\|_{\mathbb{Q}} \mu_{r}^{x}(\mathrm{~d} w)\right)^{2} \\
& \leq c\left(1+\|x\|_{\mathbb{Q}}^{2}+\mathbb{E}\left\|v^{x}(r ; s, y)\right\|_{\mathbb{Q}}^{2}\right) \\
& \leq c\left(1+\|x\|_{\mathbb{Q}}^{2}+e^{-2 \delta(r-s)}\|y\|_{\mathbb{Q}}^{2}\right),
\end{aligned}
$$

and according to (100), we get

$$
\begin{aligned}
\mathbb{E}\left|P_{r, t}^{x} \psi^{x} B_{1}\left(r, v^{x}(r ; s, y)\right)\right|^{2} & =\mathbb{E}\left\|P_{r, t}^{x}\left[B_{1}\left(r, x, v^{x}(r ; s, y)\right)-\int_{\mathbb{Q}} B_{1}(r, x, w) \mu_{r}^{x}(\mathrm{~d} w)\right]\right\|_{\mathbb{Q}}^{2} \\
& =\mathbb{E}\left\|B_{1}\left(t, x, v^{x}(t ; s, y)-B_{1}\left(t, x, \eta^{x}(t)\right)\right)\right\|_{\mathbb{Q}}^{2} \\
& \leq c \mathbb{E}\left\|v^{x}(t ; s, y)-\eta^{x}(t)\right\|_{\mathbb{Q}}^{2} \leq c e^{-\delta_{2}(t-s)}\left(1+\|x\|_{\mathbb{Q}}^{2}+\|y\|_{\mathbb{Q}}^{2}\right) .
\end{aligned}
$$

Let $\delta=\delta_{2} / 2$, it follows

$$
\begin{aligned}
\mathbb{E} \| & \frac{1}{T} \int_{s}^{s+T}\left[B_{1}\left(t, x, v^{x}(t ; s, y)\right)-\int_{\mathbb{Q}} B_{1}(t, x, w) \mu_{t}^{x}(\mathrm{~d} w)\right] \mathrm{d} t \|_{\mathbb{Q}} \\
& \leq \frac{c}{T}\left(1+\|x\|_{\mathbb{Q}}+\|y\|_{\mathbb{Q}}\right)\left(\int_{s}^{s+T} \int_{r}^{s+T} e^{-\delta(t-s)} \mathrm{d} t \mathrm{~d} r\right)^{1 / 2} \\
& \leq \frac{c}{T}\left(1+\|x\|_{\mathbb{Q}}+\|y\|_{\mathbb{Q}}\right) .
\end{aligned}
$$

Since the family of functions (131) is uniformly almost periodic, according to Theorem 3.4 in [25], we can get that the limit

$$
\lim _{T \longrightarrow \infty} \frac{1}{T} \int_{s}^{s+T} \int_{\mathbb{\square}} B_{1}(t, x, w) \mu_{t}^{x}(\mathrm{~d} w) \mathrm{d} t,
$$

converges to $\bar{B}_{1}(x)$ uniformly with respect to $s \in \mathbb{R}$ and $x \in \mathbb{K}(\mathbb{K}$ is a compact set in $\mathbb{H})$. Therefore, if we define

$$
\alpha(T, x)=\left\|\frac{1}{T} \int_{s}^{s+T} \int_{\mathbb{U}} B_{1}(t, x, w) \mu_{t}^{x}(\mathrm{~d} w) \mathrm{d} t-\bar{B}_{1}(x)\right\|_{\mathbb{\sharp}},
$$

we get the conclusion (142). Moreover, thanks to the assumption (A3), equations (117), and (139), we also can get

$$
\begin{aligned}
\alpha(T, x) \leq & \left|\frac{1}{T} \int_{s}^{s+T} \int_{\mathbb{G}}\left\|B_{1}(t, x, w)\right\|_{\mathbb{\sharp}} \mu_{t}^{x}(\mathrm{~d} w) \mathrm{d} t\right| \\
& +\left\|\bar{B}_{1}(x)\right\|_{\mathbb{\sharp}} \leq c\left(1+\|x\|_{\mathbb{Q}}\right) .
\end{aligned}
$$

Hence, the proof is complete. 
Now, we introduce the averaged equation:

$$
\begin{aligned}
\mathrm{d} u(t)= & {\left[A_{1}(t) u(t)+\bar{B}_{1}(u(t))\right] \mathrm{d} t+F_{1}(t, u(t)) \mathrm{d} w^{Q_{1}}(t) } \\
& +\int_{\mathbb{Z}} G_{1}(t, u(t), z) \tilde{N}_{1}(\mathrm{~d} t, \mathrm{~d} z), \quad u(0)=x \in \mathbb{H} .
\end{aligned}
$$

Due to assumption (A3), we can easily get that the mapping $\bar{B}_{1}$ is Lipschitz continuous. So, for any $x \in \mathbb{H}, T>0$ and $p \geq 1$, equation (151) admits a unique mild solution

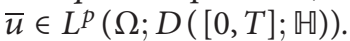

\section{Averaging Principles}

In this section, we prove that the slow motion $u_{\epsilon}$ converges to the averaged motion $\bar{u}$, as $\epsilon \longrightarrow 0$.

Theorem 1. Under (A1)-(A5), fixing $x \in \mathscr{D}\left(\left(-A_{1}\right)^{\theta}\right)$ $(\theta \in[0, \bar{\theta}))$ and $y \in \mathbb{H}$, for any $T>0$ and $\eta>0$, we have

$$
\lim _{\epsilon \longrightarrow 0} \mathbb{P}\left(\sup _{t \in[0, T]}\left\|u_{\epsilon}(t)-\bar{u}(t)\right\|_{\mathbb{G}}>\eta\right)=0,
$$

where $\bar{u}$ is the solution of the averaged (151).

Proof. For any $h \in \mathscr{D}\left(A_{1}\right) \cap L^{\infty}(\mathcal{O})$, we have

$$
\begin{aligned}
\left\langle u_{\epsilon}(t), h\right\rangle_{\mathbb{G}}= & \langle x, h\rangle_{\mathbb{G}}+\int_{0}^{t}\left\langle A_{1}(r) u_{\epsilon}(r), h\right\rangle_{\mathbb{Q}} \mathrm{d} r \\
& +\int_{0}^{t}\left\langle\bar{B}_{1}\left(u_{\epsilon}(r)\right), h\right\rangle_{\mathbb{W}} \mathrm{d} r \\
& +\left\langle\int_{0}^{t} F_{1}\left(r, u_{\epsilon}(r)\right) \mathrm{d} w^{Q_{1}}(r), h\right\rangle_{\mathbb{Q}} \\
& +\left\langle\int_{0}^{t} \int_{\mathbb{Z}} G_{1}\left(r, u_{\epsilon}(r), z\right) \tilde{N}_{1}(\mathrm{~d} r, \mathrm{~d} z), h\right\rangle_{\mathbb{W}} \\
& +R_{\epsilon}(t),
\end{aligned}
$$

where

$$
R_{\epsilon}(t):=\int_{0}^{t}\left\langle B_{1}\left(r, u_{\epsilon}(r), v_{\epsilon}(r)\right)-\bar{B}_{1}\left(u_{\epsilon}(r)\right), h\right\rangle_{\mathbb{\square}} \mathrm{d} r .
$$

According to the proof in Section 3, we know that the family $\left\{\mathscr{L}\left(u_{\epsilon}\right)\right\}_{\epsilon \in(0,1]}$ is tight in $\mathscr{P}(D([0, T] ; \mathbb{H}))$. Hence, in order to prove Theorem 1, it is sufficient to prove $\lim _{\epsilon \longrightarrow 0} \mathbb{E s u p}_{t \in[0, T]}\left|R_{\epsilon}(t)\right|=0$.

For any $\epsilon>0$ and some deterministic constant $\delta_{\epsilon}>0$, we divide the interval $[0, T]$ in subintervals of the size $\delta_{\epsilon}$. In each time interval $\left[k \delta_{\epsilon},(k+1) \delta_{\epsilon}\right], k=0,1, \ldots,\left\lfloor T / \delta_{\epsilon}\right\rfloor$, we define the following auxiliary fast motion $\widehat{v}_{\epsilon}$ :

$$
\begin{aligned}
\mathrm{d} \widehat{v}_{\epsilon}(t)= & \frac{1}{\epsilon}\left[\left(A_{2}(t)-\alpha\right) \widehat{v}_{\epsilon}(t)+B_{2}\left(t, u_{\epsilon}\left(k \delta_{\epsilon}\right), \widehat{v}_{\epsilon}(t)\right)\right] \mathrm{d} t \\
& +\frac{1}{\sqrt{\epsilon}} F_{2}\left(t, u_{\epsilon}\left(k \delta_{\epsilon}\right), \widehat{v}_{\epsilon}(t)\right) \mathrm{d} \omega^{Q_{2}}(t) \\
& +\int_{\mathbb{Z}} G_{2}\left(t, u_{\epsilon}\left(k \delta_{\epsilon}\right), \widehat{v}_{\epsilon}(t)\right) \widetilde{N}_{2}^{\epsilon}(\mathrm{d} t, \mathrm{~d} z) .
\end{aligned}
$$

According to the definition of $\widehat{v}_{\epsilon}$, we know that an analogous estimate to (31) holds. So, for any $p \geq 1$, we have

$$
\int_{0}^{T} \mathbb{E}\left\|\widehat{v}_{\epsilon}(t)\right\|_{\mathbb{Q}}^{p} \mathrm{~d} t \leq c_{p, T}\left(1+\|x\|_{\mathbb{Q}}^{p}+\|y\|_{\mathbb{Q}}^{p}\right) .
$$

Lemma 9. Under (A1)-(A5), fixing $x \in \mathscr{D}\left(\left(-A_{1}\right)^{\theta}\right)$ $(\theta \in t[n 0 q, h \bar{\theta}))$ and $y \in \mathbb{H}$, there exists a constant $\kappa>0$, such that if

$$
\delta_{\epsilon}=\epsilon \ln ^{\epsilon^{-\kappa}},
$$

we have

$$
\lim _{\epsilon \longrightarrow 0} \sup _{t \in[0, T]} \mathbb{E}\left\|\widehat{v}_{\epsilon}(t)-v_{\epsilon}(t)\right\|_{\llbracket}^{p}=0 .
$$

Proof. Let $\epsilon>0$ be fixed. For $k=0,1, \ldots,\left\lfloor T / \delta_{\epsilon}\right\rfloor$ and $t \in\left[k \delta_{\epsilon},(k+1) \delta_{\epsilon}\right]$, let $\rho_{\epsilon}(t)$ be the solution of the problem

$$
\begin{aligned}
\mathrm{d} \rho_{\epsilon}(t)= & \frac{1}{\epsilon}\left(A_{2}(t)-\alpha\right) \rho_{\epsilon}(t) \mathrm{d} t+\frac{1}{\sqrt{\epsilon}} K_{\epsilon}(t) \mathrm{d} \omega^{Q_{2}}(t) \\
& +\int_{\mathbb{Z}} H_{\epsilon}(t, z) \widetilde{N}_{2}^{\epsilon}(\mathrm{d} t, \mathrm{~d} z), \quad \rho_{\epsilon}\left(k \delta_{\epsilon}\right)=0,
\end{aligned}
$$

where

$$
\begin{aligned}
K_{\epsilon}(t) & :=F_{2}\left(t, u_{\epsilon}\left(k \delta_{\epsilon}\right), \widehat{v}_{\epsilon}(t)\right)-F_{2}\left(t, u_{\epsilon}(t), v_{\epsilon}(t)\right), \\
H_{\epsilon}(t, z) & :=G_{2}\left(t, u_{\epsilon}\left(k \delta_{\epsilon}\right), \widehat{v}_{\epsilon}(t), z\right)-G_{2}\left(t, u_{\epsilon}(t), v_{\epsilon}(t), z\right) .
\end{aligned}
$$

We get

$$
\rho_{\epsilon}(t)=\psi_{\alpha, \epsilon, 2}\left(\rho_{\epsilon} ; k \delta_{\epsilon}\right)(t)+\Gamma_{\epsilon}(t)+\Psi_{\epsilon}(t), \quad t \in\left[k \delta_{\epsilon},(k+1) \delta_{\epsilon}\right],
$$

where

$$
\begin{aligned}
& \Gamma_{\epsilon}(t)=\frac{1}{\sqrt{\epsilon}} \int_{k \delta_{\epsilon}}^{t} U_{\alpha, \epsilon, 2}(t, r) K_{\epsilon}(r) \mathrm{d} w^{Q_{2}}(r), \\
& \Psi_{\epsilon}(t)=\int_{k \delta_{\epsilon}}^{t} \int_{\mathbb{Z}} U_{\alpha, \epsilon, 2}(t, r) H_{\epsilon}(r, z) \tilde{N}_{2}^{\epsilon}(\mathrm{d} r, \mathrm{~d} z) .
\end{aligned}
$$

If we denote $\Lambda_{\epsilon}(t):=\widehat{v}_{\epsilon}(t)-v_{\epsilon}(t)$ and $\vartheta_{\epsilon}(t):=$ $\Lambda_{\epsilon}(t)-\rho_{\epsilon}(t)$, we have

$$
\begin{aligned}
\mathrm{d} \vartheta_{\epsilon}(t)= & \frac{1}{\epsilon}\left[\left(A_{2}(t)-\alpha\right) \vartheta_{\epsilon}(t)+B_{2}\left(t, u_{\epsilon}\left(k \delta_{\epsilon}\right), \widehat{v}_{\epsilon}(t)\right)\right. \\
& \left.-B_{2}\left(t, u_{\epsilon}(t), v_{\epsilon}(t)\right)\right] \mathrm{d} t .
\end{aligned}
$$

Due to Lemma 3, for $\alpha>0$ large enough, using Young's inequality, we have 


$$
\begin{aligned}
& \frac{1}{p} \frac{\mathrm{d}}{\mathrm{d} t}\left\|\vartheta_{\epsilon}(t)\right\|_{\mathbb{H}}^{p} \leq \frac{1}{\epsilon}\left\langle\left(\gamma_{2}(t) A_{2}+L_{2}(t)-\alpha\right) \vartheta_{\epsilon}(t), \vartheta_{\epsilon}(t)\right\rangle_{\mathbb{H}}\left\|\vartheta_{\epsilon}(t)\right\|_{\mathbb{H}}^{p-2}+\frac{1}{\epsilon}\left\langle B_{2}\left(t, u_{\epsilon}\left(k \delta_{\epsilon}\right), \widehat{v}_{\epsilon}(t)\right)-B_{2}\left(t, u_{\epsilon}(t), v_{\epsilon}(t)\right), \vartheta_{\epsilon}(t)\right\rangle_{\mathbb{H}}\left\|\vartheta_{\epsilon}(t)\right\|_{\mathbb{H}}^{p-2}
\end{aligned}
$$

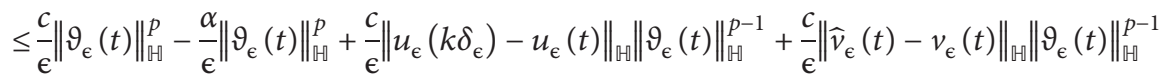

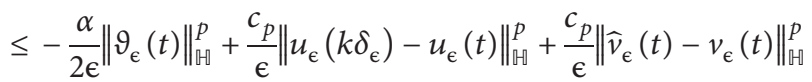

$$
\begin{aligned}
& \leq-\frac{\alpha}{2 \epsilon}\left\|\vartheta_{\epsilon}(t)\right\|_{\mathscr{H}}^{p}+\frac{c_{p}}{\epsilon}\left(1+\|x\|_{\theta}^{p}+\|y\|_{\uplus}^{p}\right)\left(\delta_{\epsilon}^{\beta(\theta) p}+\delta_{\epsilon}\right)+\frac{c_{P}}{\epsilon}\left\|\widehat{v}_{\epsilon}(t)-v_{\epsilon}(t)\right\|_{H}^{p} .
\end{aligned}
$$

Using the Gronwall inequality, we get

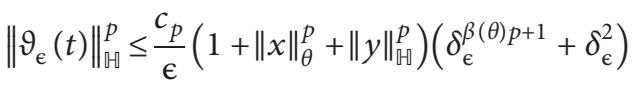

$$
\begin{aligned}
& +\frac{c_{p}}{\epsilon} \int_{k \delta_{\epsilon}}^{t}\left\|\widehat{v}_{\epsilon}(r)-v_{\epsilon}(r)\right\|_{\mathbb{H}}^{p} \mathrm{~d} r .
\end{aligned}
$$

By proceeding as Lemma 6.3 in [13], we prove that

$$
\begin{gathered}
\mathbb{E}\left\|\Gamma_{\epsilon}(t)\right\|_{\leftrightarrow \in}^{p} \leq \frac{c_{p}}{\epsilon}\left(1+\|x\|_{\theta}^{p}+\|y\|_{\mathbb{H}}^{p}\right)\left(\delta_{\epsilon}^{\beta(\theta) p+1}+\delta_{\epsilon}^{2}\right) \\
+\frac{c_{p}}{\epsilon} \int_{k \delta_{\epsilon}}^{t} \mathbb{E}\left\|\widehat{v}_{\epsilon}(r)-v_{\epsilon}(r)\right\|_{\mathbb{H}}^{p} \mathrm{~d} r .
\end{gathered}
$$

For the other stochastic term $\Psi_{\epsilon}(t)$, using Kunita's first inequality and the Hölder inequality, we get

$$
\begin{aligned}
& \mathbb{E}\left\|\Psi_{\epsilon}(t)\right\|_{\mathbb{H}}^{p} \leq c_{p} \mathbb{E}\left(\frac{1}{\epsilon} \int_{k \delta_{\epsilon}}^{t} \int_{\mathbb{Z}}\left\|e^{-(\alpha / \epsilon)(t-r)} e^{\left(\gamma_{2}(t, r) / \epsilon\right) A_{2}} H_{\epsilon}(r, z)\right\|_{\mathbb{H}}^{2} \nu_{2}(\mathrm{~d} z) \mathrm{d} r\right)^{p / 2} \\
& +\frac{c_{p}}{\epsilon} \mathbb{E} \int_{k \delta_{\epsilon}}^{t} \int_{\mathbb{Z}}\left\|e^{-\alpha / \epsilon(t-r)} e^{A_{2} \gamma_{2}(t, r) / \epsilon} H_{\epsilon}(r, z)\right\|_{\mathbb{H}}^{p} v_{2}(\mathrm{~d} z) \mathrm{d} r \\
& \leq \frac{c_{p}}{\epsilon^{p / 2}} \mathbb{E}\left(\int_{k \delta_{\epsilon}}^{t} e^{-(2 \alpha / \epsilon)(t-r)}\left(\left\|u_{\epsilon}\left(k \delta_{\epsilon}\right)-u_{\epsilon}(r)\right\|_{\mathbb{H}}^{2}+\left\|\widehat{v}_{\epsilon}(r)-v_{\epsilon}(r)\right\|_{\mathbb{H}}^{2}\right) \mathrm{d} r\right)^{p / 2} \\
& +\frac{c_{p}}{\epsilon} \int_{k \delta_{\epsilon}}^{t} \mathbb{E}\left\|u_{\epsilon}\left(k \delta_{\epsilon}\right)-u_{\epsilon}(r)\right\|_{\mathbb{H}}^{p}+\mathbb{E}\left\|\widehat{v}_{\epsilon}(r)-v_{\epsilon}(r)\right\|_{\mathbb{H}}^{p} \mathrm{~d} r \\
& \leq \frac{c_{p}}{\epsilon^{p / 2}} \int_{k \delta_{\epsilon}}^{t}\left(\mathbb{E}\left\|u_{\epsilon}\left(k \delta_{\epsilon}\right)-u_{\epsilon}(r)\right\|_{\mathbb{W}}^{p}+\mathbb{E}\left\|\widehat{v}_{\epsilon}(r)-v_{\epsilon}(r)\right\|_{\mathbb{W}}^{p}\right) \mathrm{d} r\left(\int_{k \delta_{\epsilon}}^{t} e^{-(2 \alpha / \epsilon)(p / p-2)(t-r)} \mathrm{d} r\right)^{(p-2) / 2} \\
& +\frac{c_{p}}{\epsilon} \int_{k \delta_{\epsilon}}^{t}\left(\mathbb{E}\left\|u_{\epsilon}\left(k \delta_{\epsilon}\right)-u_{\epsilon}(r)\right\|_{\mathbb{H}}^{p}+\mathbb{E}\left\|\widehat{v}_{\epsilon}(r)-v_{\epsilon}(r)\right\|_{\mathbb{H}}^{p}\right) \mathrm{d} r \\
& \leq \frac{c_{p}}{\epsilon} \int_{k \delta_{\epsilon}}^{t}\left(\mathbb{E}\left\|u_{\epsilon}\left(k \delta_{\epsilon}\right)-u_{\epsilon}(r)\right\|_{\mathbb{H}}^{p}+\mathbb{E}\left\|\hat{v}_{\epsilon}(r)-v_{\epsilon}(r)\right\|_{\mathbb{H}}^{p}\right) \mathrm{d} r \\
& \leq \frac{c_{p}}{\epsilon}\left(1+\|x\|_{\theta}^{p}+\|y\|_{\mathbb{H}}^{p}\right)\left(\delta_{\epsilon}^{\beta(\theta) p+1}+\delta_{\epsilon}^{2}\right)+\frac{c_{p}}{\epsilon} \int_{k \delta_{\epsilon}}^{t} \mathbb{E}\left\|\widehat{v}_{\epsilon}(r)-v_{\epsilon}(r)\right\|_{\mathbb{H}}^{p} \mathrm{~d} r .
\end{aligned}
$$

According to Lemma 2.4 in [25] and equations (161)-(167), we obtain

$$
\begin{aligned}
& \mathbb{E}\left\|\widehat{v}_{\epsilon}(t)-v_{\epsilon}(t)\right\|_{\mathbb{H}}^{p} \leq \frac{c_{p}}{\epsilon}\left(1+\|x\|_{\theta}^{p}+\|y\|_{\mathbb{H}}^{p}\right)\left(\delta_{\epsilon}^{\beta(\theta) p+1}+\delta_{\epsilon}^{2}\right) \\
& +\frac{c_{p}}{\epsilon} \int_{k \delta_{\epsilon}}^{t} \mathbb{E}\left\|\hat{v}_{\epsilon}(r)-v_{\epsilon}(r)\right\|_{\mathbb{H}}^{p} \mathrm{~d} r .
\end{aligned}
$$

From the Gronwall lemma, this means $\mathbb{E}\left\|\hat{v}_{\epsilon}(t)-v_{\epsilon}(t)\right\|_{\mathbb{H}}^{p} \leq \frac{c_{p}}{\epsilon}\left(\delta_{\epsilon}^{\beta(\theta) p+1}+\delta_{\epsilon}^{2}\right) e^{\left(c_{p} / \epsilon\right) \delta_{\epsilon}}\left(1+\|x\|_{\theta}^{p}+\|y\|_{\sharp}^{p}\right)$.
For $t \in[0, T]$, selecting $\delta_{\epsilon}=\epsilon \ln ^{\epsilon^{-k}}$, then if we take $\kappa<\beta(\theta) p /\left(\beta(\theta) p+1+c_{p}\right) \wedge 1 /\left(2+c_{p}\right)$, we have (158).

Lemma 10. Under the same assumptions as in Theorem 1, for any $T>0$, we have

$$
\lim _{\epsilon \longrightarrow 0} \mathbb{E} \sup _{t \in[0, T]}\left|R_{\epsilon}(t)\right|=0 .
$$

Proof. According to the definition of $\bar{B}_{1}$, we get that the

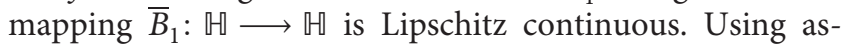
sumption (A3) and Lemmas 3 and 9, we have 


$$
\begin{aligned}
& \lim _{\epsilon \longrightarrow 0} \mathbb{E} \sup _{t \in[0, T]}\left|R_{\epsilon}(t)\right| \leq \lim _{\epsilon \longrightarrow 0} \mathbb{E} \int_{0}^{T}\left|\left\langle B_{1}\left(r, u_{\epsilon}(r), v_{\epsilon}(r)\right)-B_{1}\left(r, u_{\epsilon}\left(\left\lfloor r / \delta_{\epsilon}\right\rfloor \delta_{\epsilon}\right), \widehat{v}_{\epsilon}(r)\right), h\right\rangle_{\mathbb{H}}\right| \mathrm{d} r \\
& +\lim _{\epsilon \longrightarrow 0} \mathbb{E} \sup _{t \in[0, T]}\left|\int_{0}^{t}\left\langle B_{1}\left(r, u_{\epsilon}\left(\left\lfloor r / \delta_{\epsilon}\right\rfloor \delta_{\epsilon}\right), \widehat{v}_{\epsilon}(r)\right)-\bar{B}_{1}\left(u_{\epsilon}(r)\right), h\right\rangle_{\mathbb{\square}} \mathrm{d} r\right| \\
& \leq \lim _{\epsilon \longrightarrow 0} c_{T}\|h\|_{\mathbb{G}}\left[\sup _{r \in[0, T]} \mathbb{E}\left\|u_{\epsilon}(r)-u_{\epsilon}\left(\left\lfloor r / \delta_{\epsilon}\right\rfloor \delta_{\epsilon}\right)\right\|_{\mathbb{G}}+\sup _{r \in[0, T]} \mathbb{E}\left\|\widehat{v}_{\epsilon}(r)-v_{\epsilon}(r)\right\|_{\mathbb{G}}\right] \\
& +\lim _{\epsilon \longrightarrow 0} \sum_{k=0}^{\left\lfloor T / \delta_{\epsilon}\right\rfloor} \mathbb{E}\left|\int_{k \delta_{\epsilon}}^{(k+1) \delta_{\epsilon}}\left\langle B_{1}\left(r, u_{\epsilon}\left(\left\lfloor r / \delta_{\epsilon}\right\rfloor \delta_{\epsilon}\right), \widehat{v}_{\epsilon}(r)\right)-\bar{B}_{1}\left(u_{\epsilon}\left(k \delta_{\epsilon}\right)\right), h\right\rangle_{\mathbb{G}} \mathrm{d} r\right| \\
& +\lim _{\epsilon \longrightarrow 0}\|h\|_{\mathbb{\square}} \sum_{k=0}^{\left\lfloor T / \delta_{\epsilon}\right\rfloor} \int_{k \delta_{\epsilon}}^{(k+1) \delta_{\epsilon}} \mathbb{E}\left\|\bar{B}_{1}\left(u_{\epsilon}\left(k \delta_{\epsilon}\right)\right)-\bar{B}_{1}\left(u_{\epsilon}(r)\right)\right\|_{\mathbb{Q}} \mathrm{d} r \\
& \leq \lim _{\epsilon \longrightarrow 0} c_{T}\|h\|_{\mathbb{Q}}\left[\left(1+\|x\|_{\theta}+\|y\|_{\mathbb{Q}}\right)\left(\delta_{\epsilon}^{\beta(\theta)}+\delta_{\epsilon}\right)+\sup _{r \in[0, T]} \mathbb{E}\left\|\widehat{v}_{\epsilon}(r)-v_{\epsilon}(r)\right\|_{\mathbb{Q}}\right] \\
& +\lim _{\epsilon \longrightarrow 0} \sum_{k=0}^{\left\lfloor T / \delta_{\epsilon}\right\rfloor} \mathbb{E}\left|\int_{k \delta_{\epsilon}}^{(k+1) \delta_{\epsilon}}\left\langle B_{1}\left(r, u_{\epsilon}\left(k \delta_{\epsilon}\right), \widehat{v}_{\epsilon}(r)\right)-\bar{B}_{1}\left(u_{\epsilon}\left(k \delta_{\epsilon}\right)\right), h\right\rangle_{\mathbb{\square}} \mathrm{d} r\right| \\
& +\lim _{\epsilon \longrightarrow 0} c_{T}\|h\|_{\mathbb{Q}}\left(1+\|x\|_{\theta}+\|y\|_{\mathbb{U}}\right)\left(\delta_{\epsilon}^{\beta(\theta)+1}+\delta_{\epsilon}^{2}\right)\left(\left\lfloor T / \delta_{\epsilon}\right\rfloor+1\right) .
\end{aligned}
$$

So, we have to show that $\lim _{\epsilon \longrightarrow 0} \sum_{k=0}^{\left\lfloor T / \delta_{\epsilon}\right\rfloor} \mathbb{E}\left|\int_{k \delta_{\epsilon}}^{(k+1) \delta_{\epsilon}}\left\langle B_{1}\left(r, u_{\epsilon}\left(k \delta_{\epsilon}\right), \widehat{v}_{\epsilon}(r)\right)-\bar{B}_{1}\left(u_{\epsilon}\left(k \delta_{\epsilon}\right)\right), h\right\rangle_{\mathbb{\sharp}} \mathrm{d} r\right|=0$.

If we set $\zeta_{\epsilon}=\delta_{\epsilon} / \epsilon$, we have

$$
\begin{aligned}
& \mathbb{E}\left|\int_{k \delta_{\epsilon}}^{(k+1) \delta_{\epsilon}}\left\langle B_{1}\left(r, u_{\epsilon}\left(k \delta_{\epsilon}\right), \widehat{v}_{\epsilon}(r)\right)-\bar{B}_{1}\left(u_{\epsilon}\left(k \delta_{\epsilon}\right)\right), h\right\rangle_{\mathbb{\sharp}} \mathrm{d} r\right|=\mathbb{E}\left|\int_{0}^{\delta_{\epsilon}}\left\langle B_{1}\left(k \delta_{\epsilon}+r, u_{\epsilon}\left(k \delta_{\epsilon}\right), \widehat{v}_{\epsilon}\left(k \delta_{\epsilon}+r\right)\right)-\bar{B}_{1}\left(u_{\epsilon}\left(k \delta_{\epsilon}\right)\right), h\right\rangle_{\mathbb{G}} \mathrm{d} r\right| \\
& =\mathbb{E}\left|\int_{0}^{\delta_{\epsilon}}\left\langle B_{1}\left(k \delta_{\epsilon}+r, u_{\epsilon}\left(k \delta_{\epsilon}\right), \widetilde{v}^{u_{\epsilon}}\left(k \delta_{\epsilon}\right), v_{\epsilon}\left(k \delta_{\epsilon}\right)(r / \epsilon)\right)-\bar{B}_{1}\left(u_{\epsilon}\left(k \delta_{\epsilon}\right)\right), h\right\rangle_{\mathbb{t}} \mathrm{d} r\right| \\
& \quad=\delta_{\epsilon} \mathbb{E}\left|\frac{1}{\zeta_{\epsilon}} \int_{0}^{\zeta_{\epsilon}}\left\langle B_{1}\left(k \delta_{\epsilon}+\epsilon r, u_{\epsilon}\left(k \delta_{\epsilon}\right), \widetilde{v}^{u_{\epsilon}}\left(k \delta_{\epsilon}\right), v_{\epsilon}\left(k \delta_{\epsilon}\right)(r)\right)-\bar{B}_{1}\left(u_{\epsilon}\left(k \delta_{\epsilon}\right)\right), h\right\rangle_{\mathbb{H}} \mathrm{d} r\right|
\end{aligned}
$$

$$
\left\{u_{\epsilon}\left(k \delta_{\epsilon}\right): \epsilon>0, k=0, \ldots,\left\lfloor T / \delta_{\epsilon}\right\rfloor\right\}
$$

where $\widetilde{v}^{u_{\epsilon}}\left(k \delta_{\epsilon}\right), v_{\epsilon}\left(k \delta_{\epsilon}\right)(r)$ is the solution of the fast motion (76) with the initial datum given by $v_{\epsilon}\left(k \delta_{\epsilon}\right)$ and the frozen slow component given by $u_{\epsilon}\left(k \delta_{\epsilon}\right)$. In addition, the noises in (76) are independent of $u_{\epsilon}\left(k \delta_{\epsilon}\right)$ and $v_{\epsilon}\left(k \delta_{\epsilon}\right)$. According to the proof of Lemma 8, we get

$$
\begin{aligned}
& \mathbb{E}\left|\int_{k \delta_{\epsilon}}^{(k+1) \delta_{\epsilon}}\left\langle B_{1}\left(r, u_{\epsilon}\left(k \delta_{\epsilon}\right), \widehat{v}_{\epsilon}(r)\right)-\bar{B}_{1}\left(u_{\epsilon}\left(k \delta_{\epsilon}\right)\right), h\right\rangle_{\mathbb{\square}} \mathrm{d} r\right| \\
& \quad \leq \delta_{\epsilon} \frac{c}{\zeta_{\epsilon}}\left(1+\mathbb{E}\left\|u_{\epsilon}\left(k \delta_{\epsilon}\right)\right\|_{\mathbb{\square}}+\mathbb{E}\left\|v_{\epsilon}\left(k \delta_{\epsilon}\right)\right\|_{\mathbb{Q}}\right)\|h\|_{\mathbb{T}} \\
& \quad+\delta_{\epsilon}\|h\|_{\mathbb{Q}} \mathbb{E} \alpha\left(\zeta_{\epsilon}, u_{\epsilon}\left(k \delta_{\epsilon}\right)\right) .
\end{aligned}
$$

In Section 3, we proved that the family is tight. Then, by proceeding as the proof of equation (8.21) in [25], we also can get that, for any $\eta>0$, there exists a compact set $\mathbb{K}_{\eta} \subset \mathbb{H}$, such that

$$
\mathbb{E} \alpha\left(\zeta_{\epsilon}, u_{\epsilon}\left(k \delta_{\epsilon}\right)\right) \leq \sup _{x \in \mathbb{K}_{\eta}} \alpha\left(\zeta_{\epsilon}, x\right)+\sqrt{\eta} c\left(1+\|x\|_{\mathbb{Q}}+\|y\|_{\mathbb{Q}}\right) .
$$

Due to the arbitrariness of $\eta$ and according to Lemma 8, we can get (172). Furthermore, (170) holds.

Through the above proof, Theorem 1 is established.

\section{Data Availability}

No data were used to support this study. 


\section{Conflicts of Interest}

The authors declare that they have no conflicts of interest.

\section{Acknowledgments}

This work was partly supported by the National Natural Science Foundation of China under Grant no. 11772255, the Fundamental Research Funds for the Central Universities, the Research Funds for Interdisciplinary Subject of Northwestern Polytechnical University, the Shaanxi Project for Distinguished Young Scholars, the Shaanxi Provincial Key R\&D Program (nos. 2020KW-013 and 2019TD-010), and the Seed Foundation of Innovation and Creation for Graduate Students in Northwestern Polytechnical University (ZZ2018027).

\section{References}

[1] N. Bogolyubov and Y. Mitropolskii, Asymptotic Methods in the Theory of Nonlinear Oscillations, Gordon and Breach Science Publishers, New York, NY, USA, 1961.

[2] V. M. Volosov, "Averaging in systems of ordinary differential equations," Russian Mathematical Surveys, vol. 17, no. 6, pp. 1-126, 1962.

[3] J. G. Besjes, "On asymptotic methods for non-linear differential equations," Journal de Mecanique, vol. 8, p. 357, 1969.

[4] R. Khasminskii, "On the averaging principle for stochastic differential Itô equations," Kybernetika, vol. 4, pp. 260-279, 1968.

[5] D. Givon, "Strong convergence rate for two-time-scale jumpdiffusion stochastic differential systems," Multiscale Modeling \& Simulation, vol. 6, no. 2, pp. 577-594, 2007.

[6] M. Freidlin and A. Wentzell, Random Perturbations of Dynamical Systems, Springer Science and Business Media, Berlin, Heidelberg, 2012.

[7] J. Duan and W. Wang, Effective Dynamics of Stochastic Partial Differential Equations, Elsevier, Berlin, Heidelberg, 2014.

[8] W. F. Thompson, R. A. Kuske, and A. H. Monahan, "Stochastic averaging of dynamical systems with multiple time scales forced with $\alpha$-Stable noise," Multiscale Modeling \& Simulation, vol. 13, no. 4, pp. 1194-1223, 2015.

[9] Y. Xu, J. Duan, and W. Xu, "An averaging principle for stochastic dynamical systems with Lévy noise," Physica D: Nonlinear Phenomena, vol. 240, no. 17, pp. 1395-1401, 2011.

[10] Y. Xu, B. Pei, B. Pei, and R. Guo, "Stochastic averaging for slow-fast dynamical systems with fractional Brownian motion," Discrete \& Continuous Dynamical Systems-B, vol. 20, no. 7, pp. 2257-2267, 2015.

[11] Y. Xu, B. Pei, and J.-L. Wu, "Stochastic averaging principle for differential equations with non-Lipschitz coefficients driven by fractional Brownian motion," Stochastics and Dynamics, vol. 17, no. 02, p. 1750013, 2017.

[12] S. Cerrai, "A Khasminskii type averaging principle for stochastic reaction-diffusion equations," The Annals of Applied Probability, vol. 19, no. 3, pp. 899-948, 2009.

[13] S. Cerrai, "Averaging principle for systems of reaction-diffusion equations with polynomial nonlinearities perturbed by multiplicative noise," SIAM Journal on Mathematical Analysis, vol. 43, no. 6, pp. 2482-2518, 2011.

[14] W. Wang and A. J. Roberts, "Average and deviation for slowfast stochastic partial differential equations," Journal of Differential Equations, vol. 253, no. 5, pp. 1265-1286, 2012.
[15] B. Pei, Y. Xu, and G. Yin, “Averaging principles for SPDEs driven by fractional Brownian motions with random delays modulated by two-time-scale markov switching processes," Stochastics and Dynamics, vol. 18, no. 4, p. 1850023, 2018.

[16] B. Pei, Y. Xu, and J.-L. Wu, "Stochastic averaging for stochastic differential equations driven by fractional Brownian motion and standard Brownian motion," Applied Mathematics Letters, vol. 100, p. 106006, 2020.

[17] B. Pei, Y. Xu, Y. Xu, and Y. Bai, "Convergence of p-th mean in an averaging principle for stochastic partial differential equations driven by fractional Brownian motion," Discrete \& Continuous Dynamical Systems-B, vol. 25, no. 3, pp. 1141$1158,2020$.

[18] Z. Li and L. Yan, "Stochastic averaging for two-time-scale stochastic partial differential equations with fractional brownian motion," Nonlinear Analysis: Hybrid Systems, vol. 31, pp. 317-333, 2019.

[19] P. Gao, "Averaging principle for the higher order nonlinear schrödinger equation with a random fast oscillation," Journal of Statistical Physics, vol. 171, no. 5, pp. 897-926, 2018.

[20] P. Gao, "Averaging principle for stochastic Kuramoto-Sivashinsky equation with a fast oscillation," Discrete \& Continuous Dynamical Systems-A, vol. 38, no. 11, pp. 5649-5684, 2018.

[21] P. Gao, "Averaging principle for multiscale stochastic KleinGordon-Heat system," Journal of Nonlinear Science, vol. 29, no. 4, pp. 1701-1759, 2019.

[22] V. Chepyzhov and M. Vishik, Attractors for Equations of Mathematical Physics, American Mathematical Society, Providence, Rhode Island, USA, 2002.

[23] A. Carvalho, J. Langa, and J. Robinson, Attractors for InfiniteDimensional Non-autonomous Dynamical Systems, Springer Science and Business Media, Berlin, Germany, 2012.

[24] J. E. Bunder and A. J. Roberts, "Resolution of subgrid microscale interactions enhances the discretisation of nonautonomous partial differential equations," Applied Mathematics and Computation, vol. 304, pp. 164-179, 2017.

[25] S. Cerrai and A. Lunardi, "Averaging principle for nonautonomous slow-fast systems of stochastic reaction-diffusion equations: the almost periodic case," SIAM Journal on Mathematical Analysis, vol. 49, no. 4, pp. 2843-2884, 2017.

[26] D. Applebaum, Lévy Processes and Stochastic Calculus, Cambridge University Press, Cambridge, UK, 2009.

[27] J. Duan, An Introduction to Stochastic Dynamics, Cambridge University Press, Cambridge, UK, 2015.

[28] S. Peszat and J. Zabczyk, Stochastic Partial Differential Equations with Lévy Noise: An Evolution Equation Approach, Cambridge University Press, Cambridge, UK, 2007.

[29] J. Bertoin, Lévy Processes, Cambridge University Press, Cambridge, UK, 1998.

[30] X. Zhang, Y. Xu, B. Schmalfuß, and B. Pei, "Random attractors for stochastic differential equations driven by twosided Lévy processes," Stochastic Analysis and Applications, vol. 37, no. 6, pp. 1028-1041, 2019.

[31] Z. Li, L. Yan, and L. Xu, "Stepanov-like almost automorphic solutions for stochastic differential equations with Lévy noise," Communications in Statistics - Theory and Methods, vol. 47, no. 6, pp. 1350-1371, 2018.

[32] A. Fink, "Almost periodic differential equations," Lecture Notes in Mathematics, Vol. 377, Springer-Verlag, Berlin, Germany, 1974.

[33] B. Boufoussi and S. Hajji, "Successive approximation of neutral functional stochastic differential equations with 
jumps," Statistics \& Probability Letters, vol. 80, no. 5-6, pp. 324-332, 2010.

[34] J. Luo, "Fixed points and exponential stability of mild solutions of stochastic partial differential equations with delays," Journal of Mathematical Analysis and Applications, vol. 342, no. 2, pp. 753-760, 2008.

[35] J. Luo and T. Taniguchi, "The existence and uniqueness for non-Lipschitz stochastic neutral delay evolution equations driven by Poisson jumps," Stochastics and Dynamics, vol. 9, no. 1, pp. 135-152, 2009.

[36] A. Pazy, Semigroups of Linear Operators and Applications to Partial Differential Equations, Springer-Verlag, Berlin, Germany, 1983.

[37] G. D. Prato and J. Zabczyk, Stochastic Equations in Infinite Dimensions, Cambridge University Press, Cambridge, UK, 2014.

[38] L. C. Evans, Partial Differential Equations, American Mathematical Society, Cambridge, UK, 2nd edition, 2010.

[39] H. Amann, Linear and Quasilinear Parabolic Problems, Birkhaeuser, Basel, Switzerland, 1995.

[40] A. Pazy, Semigroups of Linear Operators and Applications to Partial Differential Equations, Springer Science \& Business Media, Berlin, Germany, 2010.

[41] S. Cerrai, "Stochastic reaction-diffusion systems with multiplicative noise and non-Lipschitz reaction term," Probability Theory and Related Fields, vol. 125, no. 2, pp. 271-304, 2003.

[42] B. Pei, Y. Xu, and J.-L. Wu, "Two-time-scales hyperbolicparabolic equations driven by Poisson random measures: existence, uniqueness and averaging principles," Journal of Mathematical Analysis and Applications, vol. 447, no. 1, pp. 243-268, 2017.

[43] P. Billingsley, Convergence of Probability Measures, John Wiley \& Son, Berlin, Germany, 1968.

[44] S. Cerrai, "Asymptotic behavior of systems of stochastic partial differential equations with multiplicative noise, stochastic partial differential equations and applications-vii," 2006.

[45] G. D. Prato and C. Tudor, "Periodic and almost periodic solutions for semilinear stochastic equations," Stochastic Analysis and Applications, vol. 13, pp. 13-33, 1995. 